\title{
Long-term IL-33-producing epithelial progenitor cells in chronic obstructive lung disease
}

\author{
Derek E. Byers, ${ }^{1}$ Jennifer Alexander-Brett, ${ }^{1}$ Anand C. Patel, ${ }^{1,2}$ Eugene Agapov, ${ }^{1}$ \\ Geoffrey Dang-Vu, ${ }^{1}$ Xiaohua Jin, ${ }^{1}$ Kangyun Wu, ${ }^{1}$ Yingjian You, ${ }^{1}$ Yael Alevy, ${ }^{1}$ \\ Jean-Philippe Girard, ${ }^{3}$ Thaddeus S. Stappenbeck, ${ }^{4}$ G. Alexander Patterson, ${ }^{5}$ \\ Richard A. Pierce, ${ }^{1}$ Steven L. Brody, ${ }^{1}$ and Michael J. Holtzman ${ }^{1,6}$
}

\begin{abstract}
${ }^{1}$ Department of Medicine and 2Department of Pediatrics, Washington University School of Medicine, Saint Louis, Missouri, USA. ${ }^{3}$ nnstitute of Pharmacology and Structural Biology, National Center of Scientific Research, University of Toulouse, Toulouse France. ${ }^{4}$ Department of Pathology and Immunology,

${ }^{5}$ Department of Surgery, and ${ }^{6}$ Department of Cell Biology, Washington University School of Medicine, Saint Louis, Missouri, USA.
\end{abstract}

\begin{abstract}
Chronic obstructive lung disease is characterized by persistent abnormalities in epithelial and immune cell function that are driven, at least in part, by infection. Analysis of parainfluenza virus infection in mice revealed an unexpected role for innate immune cells in IL-13-dependent chronic lung disease, but the upstream driver for the immune axis in this model and in humans with similar disease was undefined. We demonstrate here that lung levels of IL-33 are selectively increased in postviral mice with chronic obstructive lung disease and in humans with very severe chronic obstructive pulmonary disease (COPD). In the mouse model, IL-33/IL-33 receptor signaling was required for $I l 13$ and mucin gene expression, and Il33 gene expression was localized to a virus-induced subset of airway serous cells and a constitutive subset of alveolar type 2 cells that are both linked conventionally to progenitor function. In humans with COPD, IL33 gene expression was also associated with $I L 13$ and mucin gene expression, and $I L 33$ induction was traceable to a subset of airway basal cells with increased capacities for pluripotency and ATP-regulated release of IL-33. Together, these findings provide a paradigm for the role of the innate immune system in chronic disease based on the influence of long-term epithelial progenitor cells programmed for excess IL-33 production.
\end{abstract}

\section{Introduction}

It is widely believed that the innate immune system mediates the acute response to an infectious agent, but recent work shows that this response can also translate acute infection into chronic inflammatory disease. This paradigm may apply particularly to the chronic airway disease found in chronic obstructive pulmonary disease (COPD) (1). In this case, bacterial infection of the lower airways is often associated with COPD exacerbation and progression (2), but more sensitive PCR-based technology detects respiratory viruses in the airway with high frequency as well (3-7). Moreover, viral challenge shows that viral infection alone is sufficient to induce COPD exacerbation and to lead to secondary bacterial infection with exacerbation $(8,9)$. Despite these associations, a primary cause-and-effect relationship between viral infection and the pathogenesis of COPD remains to be fully established. In that regard, the relatively transient nature of most respiratory viral infections and the relatively permanent nature of chronic inflammatory lung disease remain difficult to reconcile. This discrepancy appears even more difficult to resolve for inflammation, due to an innate immune response that is conventionally viewed as built for short-term, rather than long-term, activation.

To better understand the connections among viral infection, immune response, and chronic obstructive lung disease, we devel-

Authorship note: Derek E. Byers and Jennifer Alexander-Brett contributed equally to this work.

Conflict of interest: Michael J. Holtzman is the principal investigator for grants to Washington University from Hoffman-La Roche and Forest Labs.

Citation for this article: J Clin Invest. 2013;123(9):3967-3982. doi:10.1172/JCI65570 oped a mouse model of these events and a corresponding system for analysis of COPD patients, from which whole lung explants are available for study. Our initial work on the mouse model showed that a single infection with a mouse parainfluenza virus known as Sendai virus (SeV) leads to long-term airway inflammation (10). Analysis of this model uncovered an innate immune axis involving semi-invariant NKT cells and alternatively activated (M2) macrophages that resulted in IL-13 expression and consequent airway hyperreactivity (monitored by methacholine-induced bronchoconstriction) and mucus overproduction (signified by mucin MUC5AC expression) (11). We also found initial evidence of IL-13 expression along with M2 monocyte/macrophage accumulation and MUC5AC production in the lungs of patients with severe COPD (11-13). These results identified an innate immune response to translate viral infection into chronic obstructive lung disease, but still did not explain how the response could be perpetuated.

To address this issue, we reasoned that persistent upstream events might continually drive the innate immune axis we had identified. In that regard, studies of other experimental models have revealed that the innate immune system can control IL-13 production and the associated Th2 response with at least 3 key mediators: TSLP, IL-25, and IL-33 $(14,15)$. Each of these 3 cytokines has been reported to be the product of both parenchymal cells (especially at the epithelial or endothelial surface) and various immune cells, and each has been shown to be necessary for the development of Th2 inflammation and airway hyperreactivity in experimental models of asthma using allergen challenge (16-21). Considerably less is known about these cytokines during the innate immune response to respiratory viral infection and any associated chronic lung dis- 


\section{A}
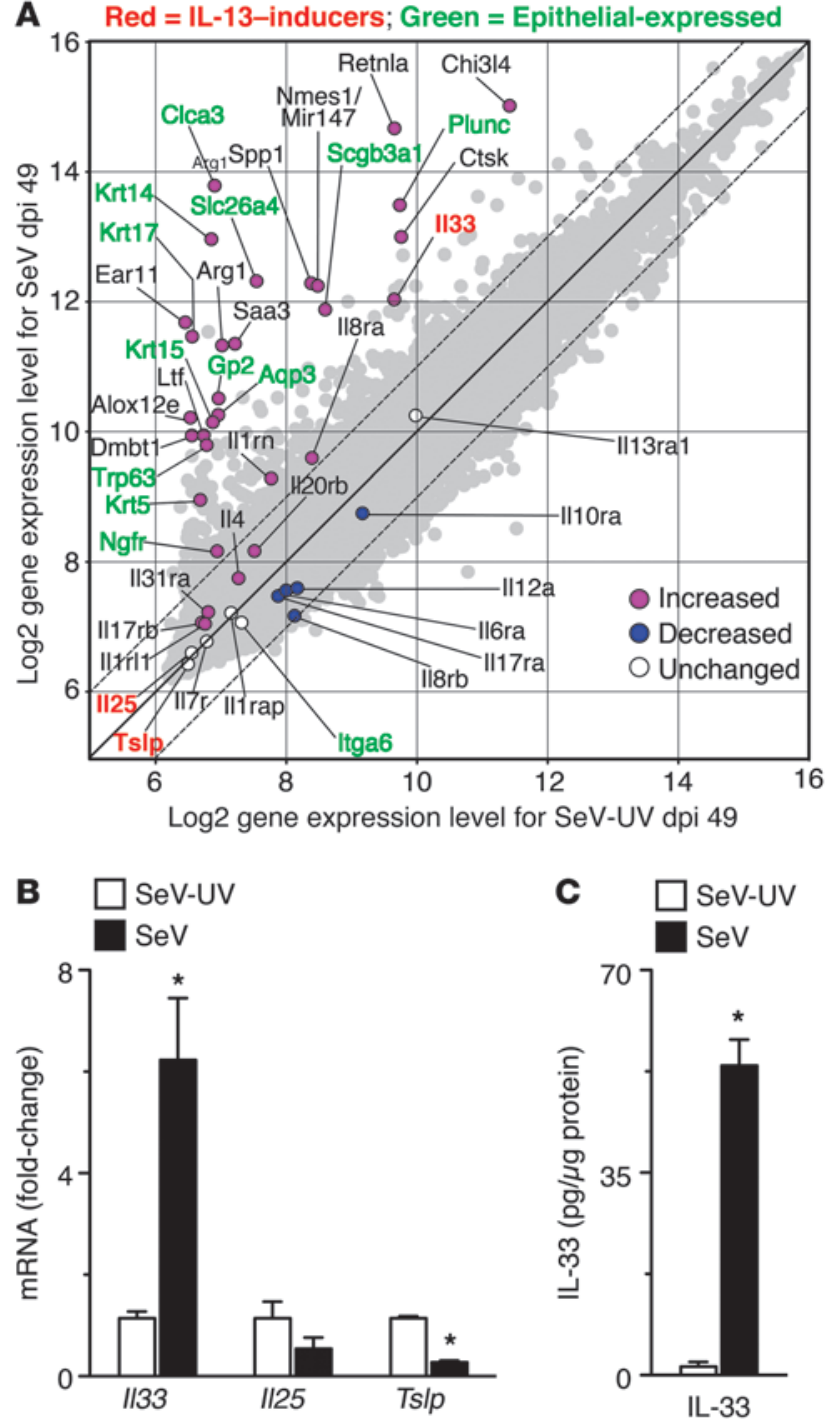
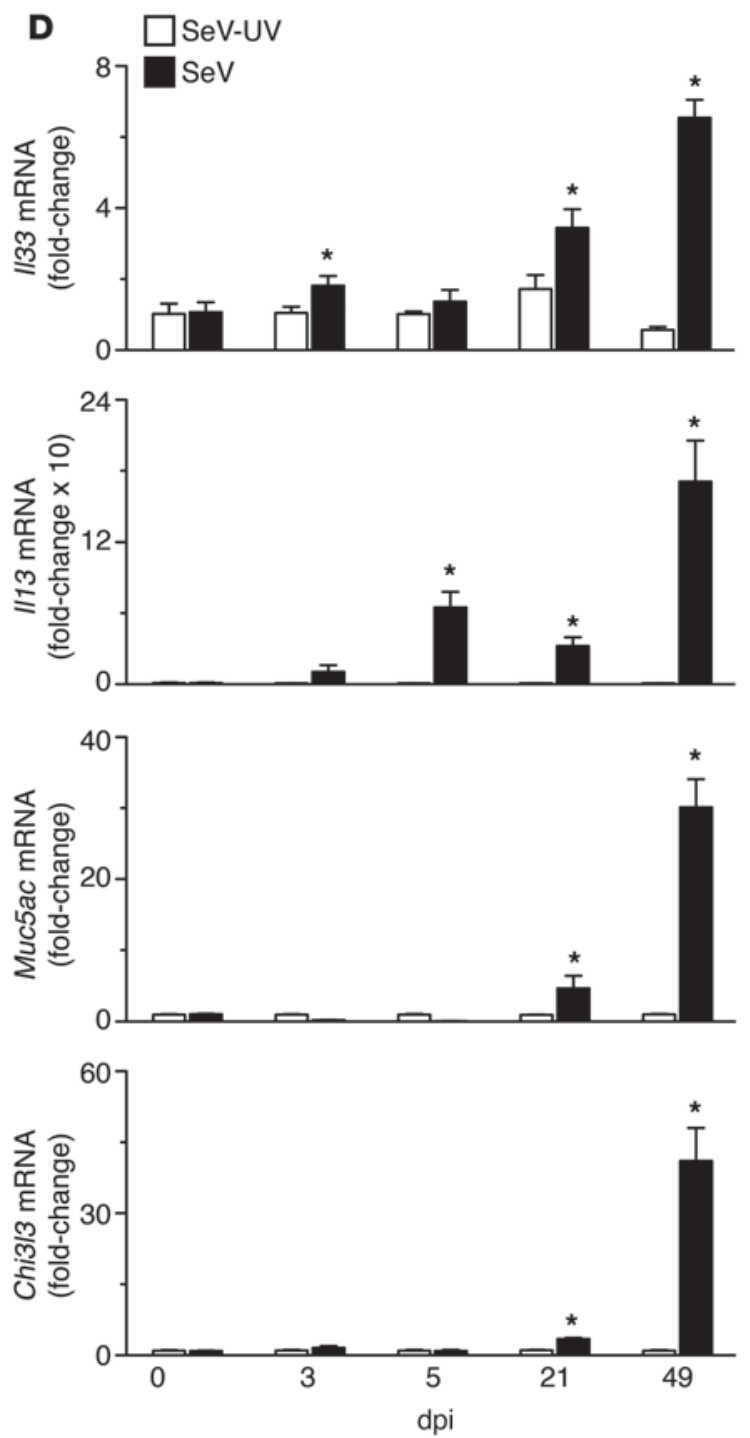

Figure 1

Selective increase in I/33 gene expression in a postviral mouse model of chronic lung disease. (A) Gene expression microarray analysis of mRNA

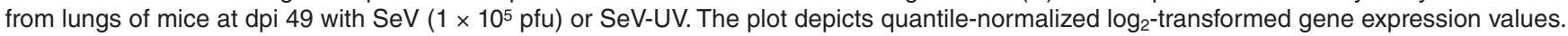
Each symbol represents the expression value for an individual gene. The 20 genes with the greatest relative change, additional relevant genes, and those encoding ILs and IL receptors are annotated. Diagonal lines represent line of equality (solid line) and 2-fold expression difference (dashed line). Significant differences for SeV versus SeV-UV values (determined as described in Methods) are indicated by colored symbols for annotated genes. (B) Expression levels of I/33, II25, and Ts/p mRNA in whole lung tissues from mice with SeV or SeV-UV at dpi 49. (C) Levels of IL-33, determined by ELISA, using lung lysates from dpi 49. (D) Levels of //33, I/13, Muc5ac, and Chi3/3 mRNA at the indicated dpi. (B-D) Values represent mean \pm SEM $\left(n=5-7\right.$ per condition). ${ }^{*} P<0.05$ versus corresponding SeV-UV.

ease. Initial work showed that IL-33 receptor (also known as ST2) signaling promoted the Th2 response to respiratory syncytial virus (RSV) in RSV-G-primed mice (22), but implications for host defense or postviral disease are difficult to discern, since the replication of a human-specific pathogen such as RSV is limited in mice, and any effects on airway inflammation and dysfunction are shortlived (23). A more recent report showed that IL-33 production from lung macrophages was required for airway hyperreactivity after infection with influenza A virus (IAV) (24). However, similar to the RSV model, this study also concentrated on the early response to virus, in this case at only 1 day after infection. This outcome may not fit with the full spectrum of clinical experience in humans, in which inflammatory airway disease might last for weeks and might further progress for many years. Moreover, there is little extension of these findings in mice to humans. There are preliminary reports of increased TSLP in COPD (25) and TSLP, IL-25, and IL-33 in asthma (26-29), but the mechanism for increased expression remains uncertain, particularly as it might relate to viral infection. Moreover, there is little information on the effect of these pathways on airway mucus production, despite the primary role of this endpoint in the morbidity and mortality of obstructive lung disease (30-33).

In the present study, we examined the potential role of TSLP, IL-25, and IL-33 in the postviral mouse model and in patients with very severe COPD. In both systems, we found that IL-33 was the 
primary candidate for regulating events upstream to IL-13 production. Moreover, IL-33 production localized to types of lung epithelial cells linked to progenitor function in both mice and humans (34-36). Indeed, tools for basal cell isolation allowed us to define a subset of this population as a potentially renewable source of excess IL-33 in COPD. Together, the findings help to reconcile the acute and transient nature of the antiviral response of the innate immune system, including a new epithelial progenitor cell component, with the persistent and sometimes-progressive nature of chronic obstructive lung disease.

\section{Results}

Increased IL-33 in the mouse model. We analyzed whole genome expression in lungs from mice inoculated with $\mathrm{SeV}$ versus UV-inactivated $\mathrm{SeV}(\mathrm{SeV}-\mathrm{UV})$ and obtained the expected increase in expression of genes for mucus production (e.g., Clca3 and Muc5ac) and M2 differentiation (e.g., Alox12e, Arg1, Chit3l3/4, Mmp12, and Retnla) at 49 days postinoculation (dpi 49) (Figure 1A and Supplemental Table 1; supplemental material available online with this article; doi:10.1172/ JCI65570DS1). In addition, we found that Il33 gene expression was the most upregulated of all IL-encoding genes represented on the microarray (Figure 1A and Supplemental Table 1). In contrast, there was no significant upregulation of either Il25 or Tslp mRNA level at $\mathrm{SeV}$ dpi 49. Selective induction of Il33 mRNA was confirmed by specific real-time PCR assay of lung mRNA from mice at $\mathrm{SeV}$ dpi 49 compared with mice inoculated with SeV-UV or PBS (Figure 1B and data not shown). Moreover, postviral induction of Il33 mRNA was accompanied by a corresponding increase in the level of IL-33 protein in the lung at $\mathrm{SeV}$ dpi 49 (Figure 1C). In contrast to the pattern for induction of IL-33, the level of Il25 mRNA was again not significantly different, while the level of Tslp mRNA was significantly decreased, at SeV dpi 49 (Figure 1B). The time course for Il33 gene expression developed in concert with $\mathrm{Il13}$ gene expression, with an early increase at $\mathrm{SeV}$ dpi 3 and a later increase at dpi 21 that became maximal at dpi 49 (Figure 1D). This late increase in Il33 and Il13 gene expression corresponded to increases in IL-13 target gene expression, including Muc5ac (representative of mucus production) and Chi3l3 (representative of M2 differentiation) (Figure 1D). This time course of gene expression was not found for Il25 or Tslp (Supplemental Figure 1). Together, these findings suggested linkage between IL-33 expression and IL-13-dependent lung disease after viral infection.

Requirement for IL-33 in the mouse model. We next used 3 approaches to determine whether IL-33/IL-33 receptor signaling is required for IL-13 production and consequent chronic lung disease in the postviral mouse model. In the first approach, we blocked signaling with a $\mathrm{mAb}$ directed against IL-1 receptor-like 1 (IL1RL1), the ligand-binding component of the heterodimeric IL-33 receptor. We observed a marked decrease in the levels of IL-13+ immune cells and MUC5 $\mathrm{AC}^{+} \mathrm{PAS}^{+}$mucous cells in the lung at $\mathrm{SeV}$ dpi 49 in mice treated with anti-IL1RL1 mAb compared with untreated or control $\mathrm{IgG}_{1} \mathrm{mAb}$-treated mice (Figure $2 \mathrm{~A}$ ). These inhibitory effects translated into a corresponding decrease in induction of Il13 and Muc5ac gene expression as well as MUC5 $\mathrm{AC}^{+}$mucous cells at $\mathrm{SeV}$ dpi 49 (Figure 2, B and C). In addition, we also found significant decreases in the levels of Arg1 and Chi3l3 mRNA in the lung at $\mathrm{SeV}$ dpi 49 in mice treated with anti-IL1RL1 mAb compared with control mice (Figure 2D). These results were consistent with a decrease in M2 differentiation during postviral disease. Treatment with anti-IL1RL1 mAb was begun on SeV dpi 12, so the effect cannot be attributed to an action on the acute inflammation and/or viral replication found after $\mathrm{SeV}$ inoculation, which subside by this time (11). In contrast to the decrease in IL-13 production, we found no significant changes in Il33 or Il1rl1 mRNA levels during anti-IL-33 mAb treatment (Figure 2E). We also observed no gross difference in the degree of immune cell infiltration in anti-IL1RL1 mAb-treated mice, similar to previous results obtained with blockade of IL-13/IL-13 receptor signaling (11).

In the second approach, we examined the effect of interrupting IL-33/IL-33 receptor signaling using Illrl1 ${ }^{-/-}$mice. We detected no difference in acute illness (as assessed by weight loss, viral level, and tissue inflammatory response) between $\mathrm{Il1rl1^{-/ }}$ and WT mice after $\mathrm{SeV}$ inoculation (Figure 3, A-C). However, we again observed significant blockade of the expected increases in mucus production, IL-13 and MUC5AC immunostaining, and Il13, Muc5ac, and Arg1 mRNA at SeV dpi 49 in Il1rl1 ${ }^{-1-}$ versus WT mice (Figure 3, D-F). The Illrl1 $1^{-1-}$ mice also afforded an opportunity to determine the effect of IL-33/IL-33 receptor signaling on airway hyperreactivity after viral infection, since we found alterations in reactivity even with control antibody treatment (data not shown). In any case, we observed attenuation of postviral increases in absolute values for lung resistance at baseline and after methacholine challenge, but no significant effect on fold change in baseline resistance after methacholine challenge in Il1rl1 ${ }^{-/-}$mice (Figure 3, G and H). These results suggest that IL-33/IL-33 receptor signaling to IL-13 production does not fully control airway hyperreactivity and that additional factors may influence this response after viral infection. The findings highlight again the relatively potent control of the IL-33to-IL-13 axis over airway mucus production after viral infection.

In the third approach, we determined the effect of IL-33 deficiency on postviral response using homozygous IL-33 gene trap (Il33 $3^{\mathrm{Gt} / \mathrm{Gt}}$ ) mice, which were engineered with a $L a c Z$ reporter to disrupt the IL-33 allele (37). In this case, we again found no difference in acute illness in $I l 33^{G t / G t}$ versus WT mice (Figure 4, A-C), but observed significant blockade of the expected development of chronic disease, signified by IL-13 and mucus production and M2 differentiation (Figure 4, D-F). Together with our previous work $(11,12)$, these 3 approaches (anti-IL1RL1 mAb, IL1RL1 deficiency, and IL-33 deficiency) served to demonstrate a specific role for IL-33/IL-33 receptor signaling in driving lung IL-13 and mucus production in the postviral mouse model of chronic obstructive lung disease.

Consistent with a role for IL-33 production in IL-13-dependent disease after viral infection, we found that delivery of IL-33 to the mouse airway was also sufficient to cause IL-13 production and consequent mucus overproduction (Supplemental Figure 2). Administration of IL-25 also caused induction of Il13 and Muc5ac gene expression, but blockade of IL-25/IL-25 receptor signaling (accomplished using Il25 $5^{-/-}$and $I l 17 \mathrm{rb}^{-/-}$mice as well as by antiIL-25 and anti-IL17RB mAb administration) had no significant effect on airway inflammation, mucus overproduction, or Il13 and $M u c 5 a c$ gene induction at $\mathrm{SeV}$ dpi 49 (Supplemental Figure 2). These results, together with the lack of induction of $I l 25$ gene expression in the postviral lung (Figure 1, A and B), indicate that IL-25/IL-25 receptor signaling does not contribute significantly to IL-13 and mucus production in the postviral mouse model. These results contrast with the reported role of IL-25 in driving IL-13 production in ovalbumin and/or Aspergillus oryzae sensitizationchallenge models of allergic airway disease $(17,38)$.

IL-33-expressing epithelial cells in the mouse model. We also used 3 approaches to determine the cellular site for increased IL-33 expression in the postviral mouse model. In the first approach, FACS-based 
A
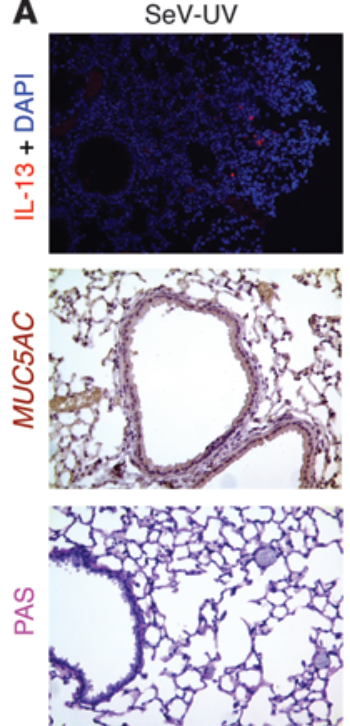

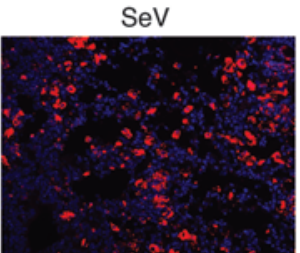

$\mathrm{SeV}+\lg \mathrm{G}$

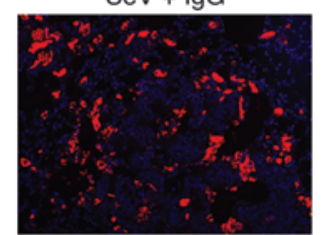

$\mathrm{SeV}+\alpha-\mathrm{IL} 1 \mathrm{RL} 1$
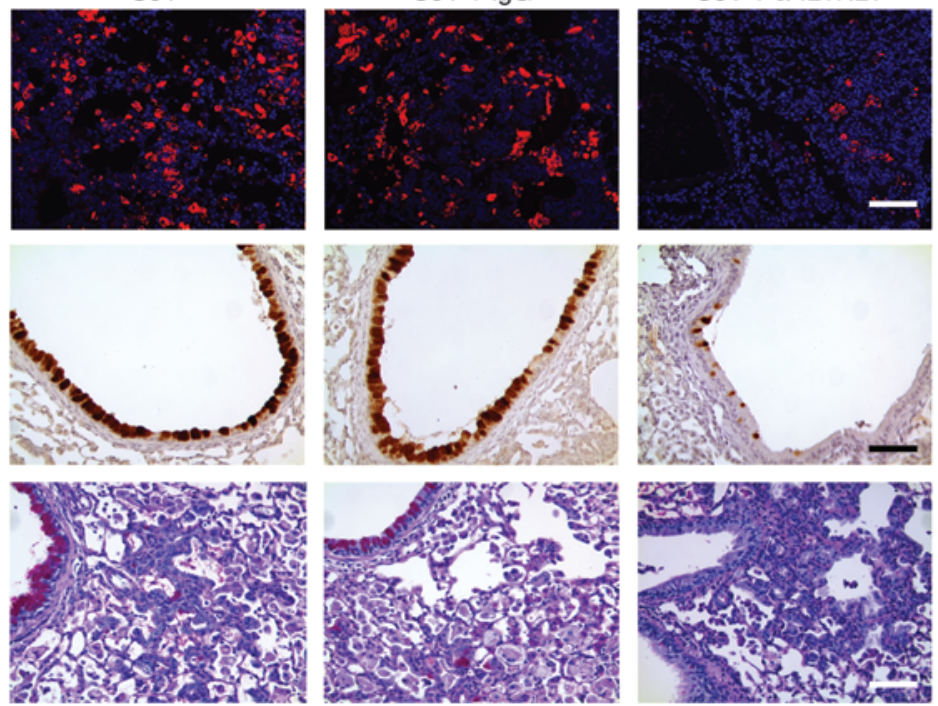

B
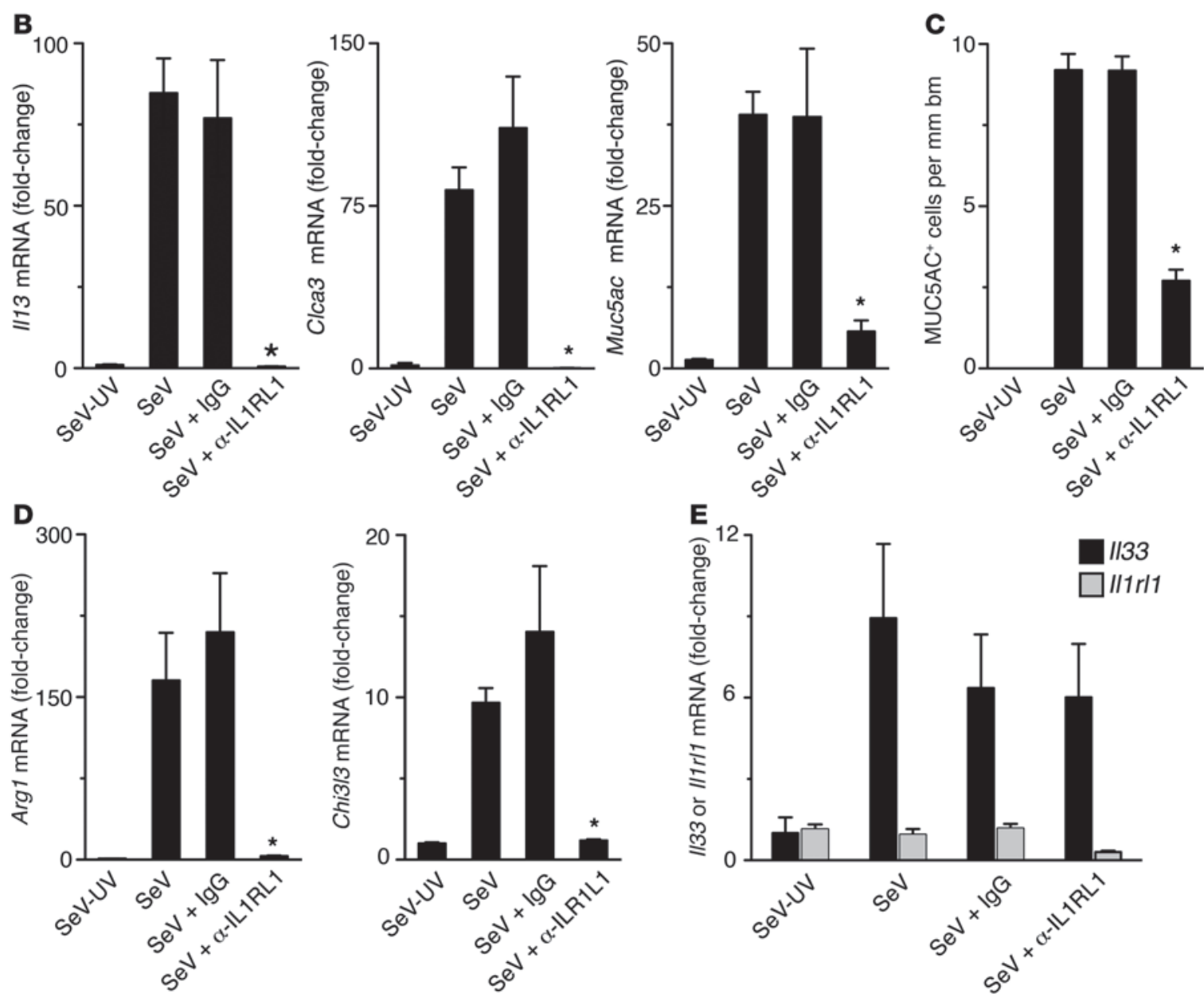

Figure 2

Effect of IL1RL1 blockade in the postviral mouse model. Mice were inoculated with SeV-UV or SeV, then treated as indicated with anti-IL1RL1 mAb or control IgG 1 mAb (IgG) on dpi 12-49, and examined at dpi 49. (A) Representative photomicrographs of mouse lung sections for IL-13 and MUC5AC immunostaining and PAS staining. Scale bars: $200 \mu \mathrm{m}$. (B) Lung levels of $/ 113$, Clca3, and Muc5ac mRNA. (C) Levels of MUC5AC+ airway epithelial cells. (D). Lung levels of Arg1 and Chi3/3 mRNA. (E) Lung levels of //33 and I/1rl1 mRNA. (B-E) Values represent mean \pm SEM ( $n=7$ per group, representative of 3 experiments). ${ }^{*} P<0.05$ versus untreated SeV. 
A

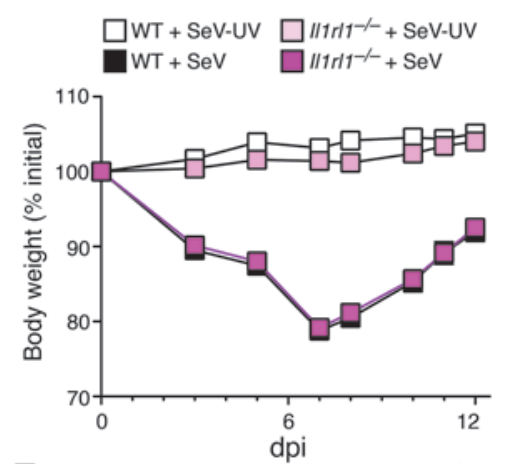

B

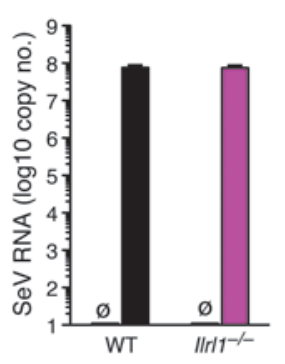

C

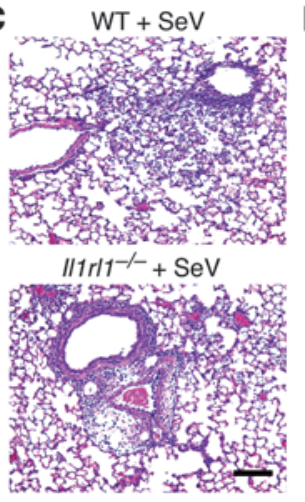

D
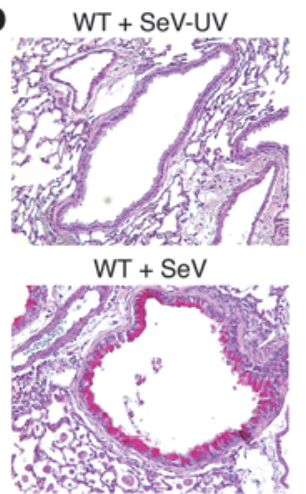

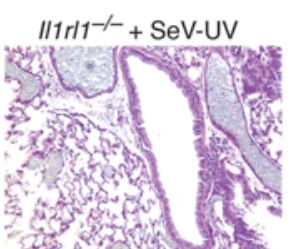

$\| 1 \mathrm{rl} 1^{-1}+\mathrm{SeV}$

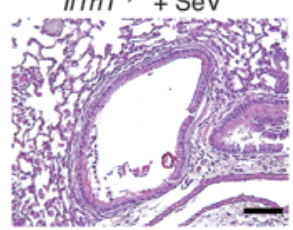

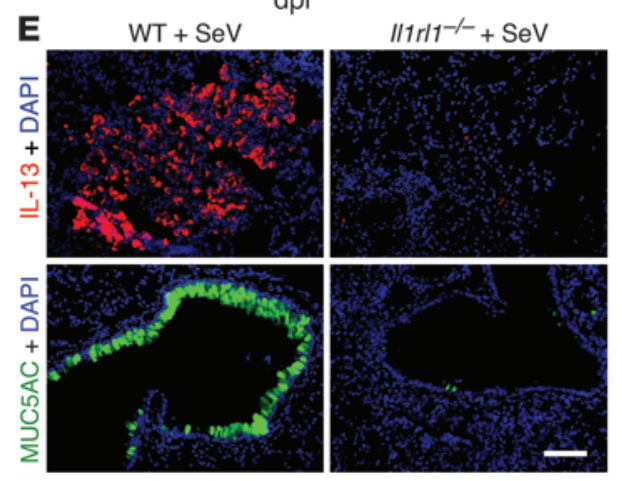
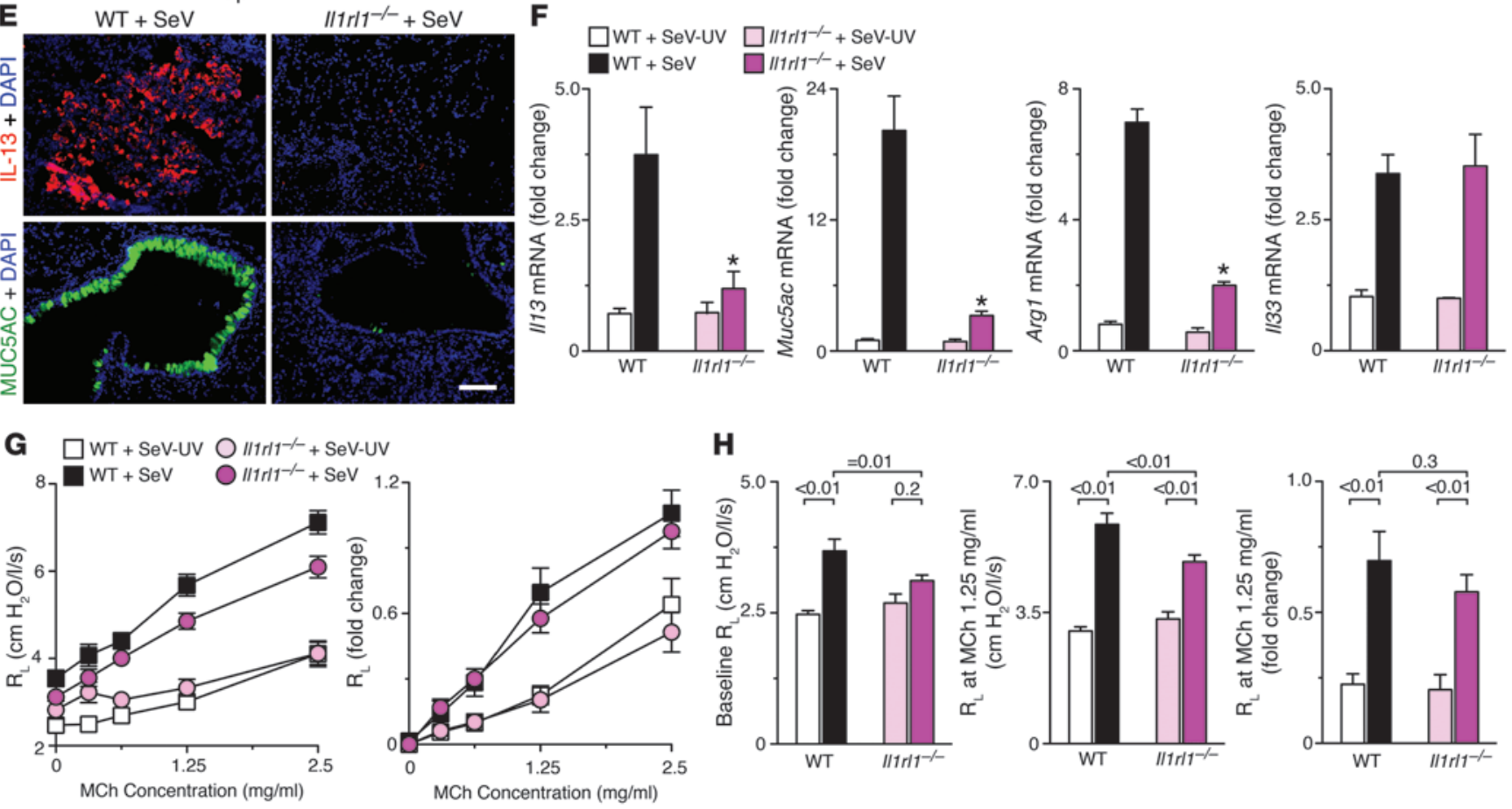

Figure 3

Effect of IL1RL1 deficiency in the postviral mouse model. //1r/1-/- and WT mice were inoculated with SeV or SeV-UV. (A) Body weight. (B) Lung levels of SeV RNA at dpi 5. (C) Representative photomicrographs showing H\&E staining of lung sections at dpi 5. (D) Representative photomicrographs showing PAS staining of lung sections at dpi 49. (E) Representative photomicrographs showing IL-13 and MUC5AC immunostaining of lung sections at dpi 49. (F) Lung levels of $/ / 13$, Muc5ac, Arg1, and $/ / 33$ mRNA ( $n=5-7$ per group). ${ }^{*}<<0.05$ versus corresponding WT. $(\mathbf{G}$ and $\mathbf{H})$ Airway reactivity to inhaled methacholine (MCh) at SeV dpi $49\left(n=15-21\right.$ mice per group). (G) Total lung resistance $\left(R_{\mathrm{L}}\right)$ and fold change from baseline were not significantly different by 2- and 3-way ANOVA. (H) Differences for lung resistance at baseline and after methacholine $(1.25 \mathrm{mg} / \mathrm{ml})$. Scale bars: $200 \mu \mathrm{m}$.

analysis of lung cell populations was used to demonstrate that the levels of Il33 mRNA were increased to the greatest extent in CD45lung cells from mice at SeV dpi 49, whether Il33 level was compared across all cell populations $\left(\mathrm{CD}_{4} 5^{-}, \mathrm{CD} 45^{+}\right.$, and $\left.\mathrm{CD} 45^{+} \mathrm{F} 4 / 80^{+}\right)$or within each individual cell population (Figure 5A and Supplemental Figure 3). The levels of Il33 mRNA copies were 600- to 100 -fold higher in the CD45- cell population compared with $\mathrm{CD} 45^{+}$hematopoietic cells, including $\mathrm{CD}^{4} 5^{+} \mathrm{F} 4 / 80^{+}$lung macrophages, at $\mathrm{SeV}$ dpi 49. Within the CD45- population, Il33 mRNA was found primarily in cells expressing EpCAM (also known as CD326), consistent with an epithelial (and perhaps progenitor/stem) cell origin (Supplemental Figure 3 and refs. 39, 40). We also detected an increase in the levels of Il33 mRNA in $\mathrm{CD} 45^{+}$cells, including $\mathrm{CD} 45^{+} \mathrm{F} 4 / 80^{+}$macrophages, at
SeV dpi 1 and 3 as well as IAV dpi 1; however, the levels of Il33 mRNA were still at least 40-fold higher in $\mathrm{CD}^{4} 5^{-}$cells, even at these early times after infection (Supplemental Figure 3).

In the second approach, we further localized the site for induction of IL-33 expression using in situ hybridization for Il33 mRNA. In this case, we again found predominant induction of Il33 gene expression in epithelial cells at $\mathrm{SeV}$ dpi 49 (Figure 5B). The most abundant Il33 mRNA signal was found in airway epithelial cells with morphology typical of serous cells (also known as Clara cells). A trace signal was also detected in cells with alveolar type 2 cell morphology and was present with and without $\mathrm{SeV}$ infection. In all cases, the Il33 mRNA signal was cytosolic, as expected for mRNA localization. 


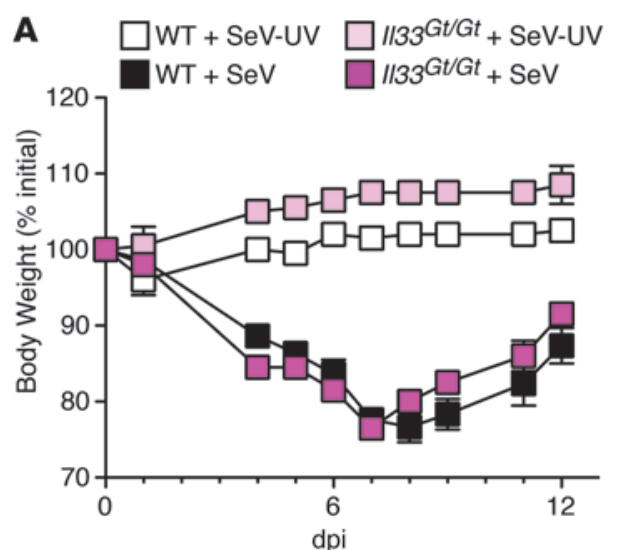

D
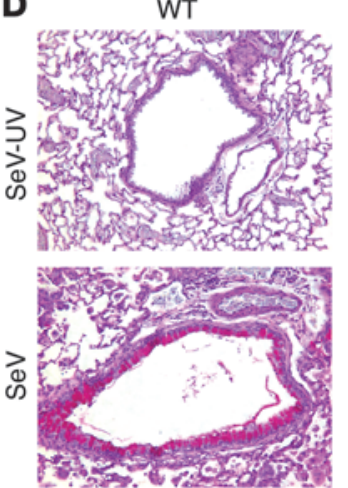

$1 / 33^{G t / G t}$
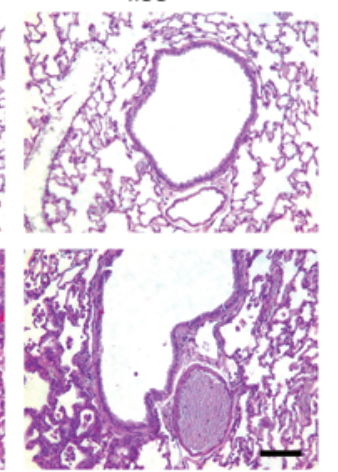

B

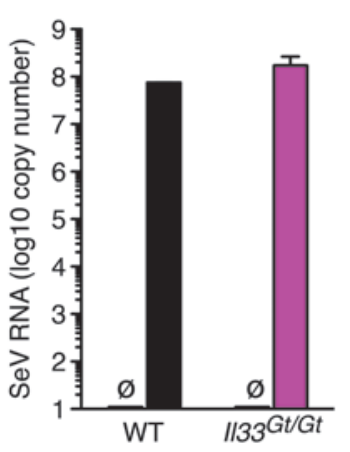

E
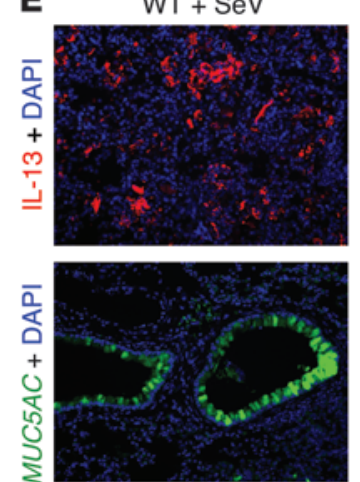

C $\quad W T+S e V$

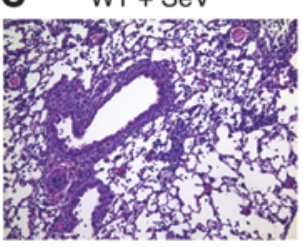

$1 / 33^{G t / G t}+\mathrm{SeV}$

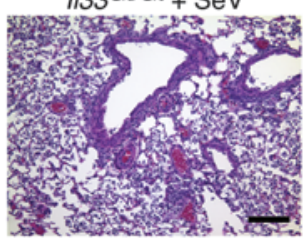

$1 / 33^{G t / G t}+\mathrm{SeV}$
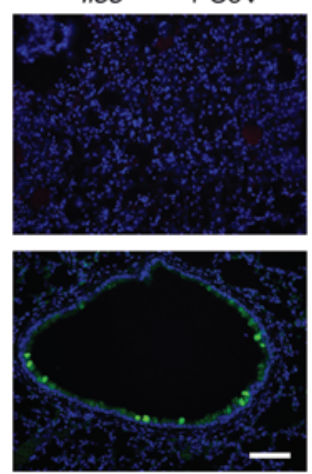

\section{Figure 4}

Effect of IL-33 deficiency in the postviral mouse model. //33 ${ }^{\mathrm{G} t / G t}$ and WT mice were inoculated with $\mathrm{SeV}$ or SeV-UV. (A) Body weight. (B) Lung levels of SeV RNA at dpi 5. (C) Representative photomicrographs showing H\&E staining of lung sections at dpi 5. (D) Representative photomicrographs showing PAS staining of lung sections at dpi 49. (E) Representative photomicrographs showing IL- 13 and MUC5AC immunostaining of lung sections at dpi 49 . (F) Lung levels of I/13, Muc5ac, Arg1, and II1rl1 mRNA ( $n=5-7$ per group). ${ }^{*} P<0.05$ versus corresponding WT. Scale bars: $200 \mu \mathrm{m}$.

\section{F $\square \mathrm{WT}+$ SeV-UV $\square \| 133^{G t / G t}+$ SeV-UV \\ $\mathrm{WT}+\mathrm{SeV} \square / 133^{G t / G t}+\mathrm{SeV}$}
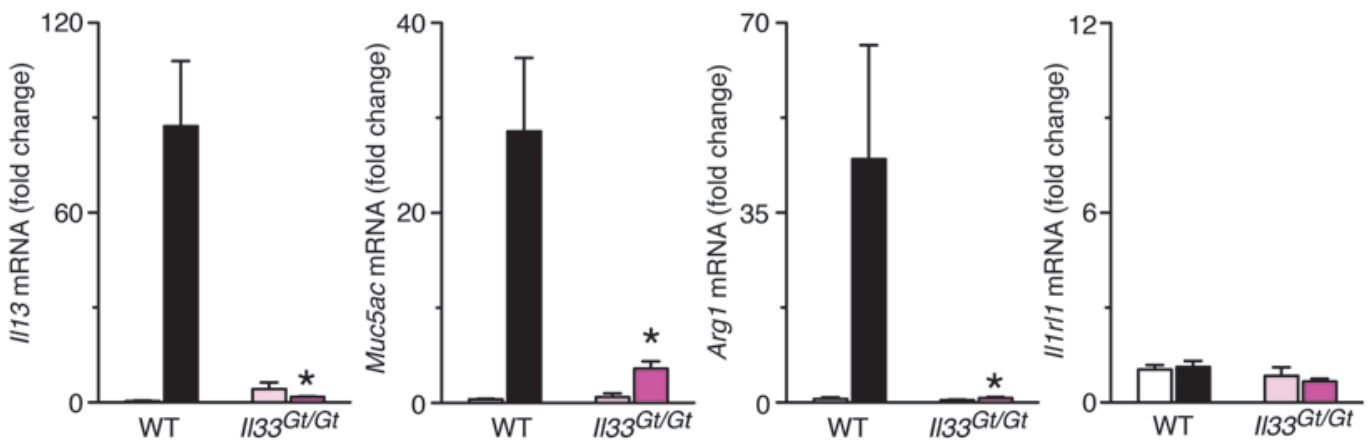

In the third approach, we used heterozygous IL-33 gene trap $\left(I l 3^{W t / G t}\right)$ mice, which express the LacZ reporter cassette under the endogenous Il33 gene promoter (37) and manifested acute and chronic responses to $\mathrm{SeV}$ infection similar to those of WT mice, including development of mucus production and IL-13 and MUC5AC immunostaining (Figure 5C). Il33 ${ }^{W t / G t}$ mice also showed induction of Il33 gene expression (signified by $\beta$-gal reporter expression) in a subset of cells with location and morphology typical of airway serous cells at $\mathrm{SeV}$ dpi 49, and these cells costained with serous cell markers secretoglobin 1a1 (SCGB1A1) and especially SCGB3A1 (Figure 5D). SCGB1A1 staining was also present at baseline conditions (SeV-UV dpi 49), but SCGB3A1 staining was more pronounced at $\mathrm{SeV}$ dpi 49. These results were consistent with whole genome expression microarray of lung tissue that showed increased mRNA for airway serous cell genes (e.g., Scgb3a1 and Cyp7b1) at SeV dpi 49 compared with SeV-UV infection (Figure 1A and Supplemental Table 1). We also detected IL-33/ $/$-gal expression in a subset of cells with location and morphology typical of alveolar type 2 cells that costained with the alveolar type 2 cell marker surfactant protein C (SFTPC), but these signals were also found at baseline, as noted previously (37).

We also detected KRT5 ${ }^{+}$basal cells in the distal airway and in the parenchyma (Figure 5E). This cell population is normally restricted to the trachea $(36,41)$, but is detectable after IAV infection in the same location as that after $\mathrm{SeV}$ infection (42). This result was also consistent with whole genome expression of lung tissue at $\mathrm{SeV}$ dpi 49 that showed significant increases in mRNA for basal cell genes (e.g., Krt5, Krt14, Krt15, Krt17, Aq3, Trp63, and Ngrr) (Figure 1A and Supplemental Table 1). However, IL-33/ $\beta$-gal expression was not detected in $\mathrm{KRT5}^{+}$basal cells, MUC5AC ${ }^{+}$mucous cells, $\alpha$-tubulin ${ }^{+}$ciliated cells, or $\mathrm{F} 4 / 80^{+}$monocytes/macrophages (Figure $5 \mathrm{E}$ and Supplemental Figure 4). IL-33/ $\beta$-gal expression was no longer detectable in WT 

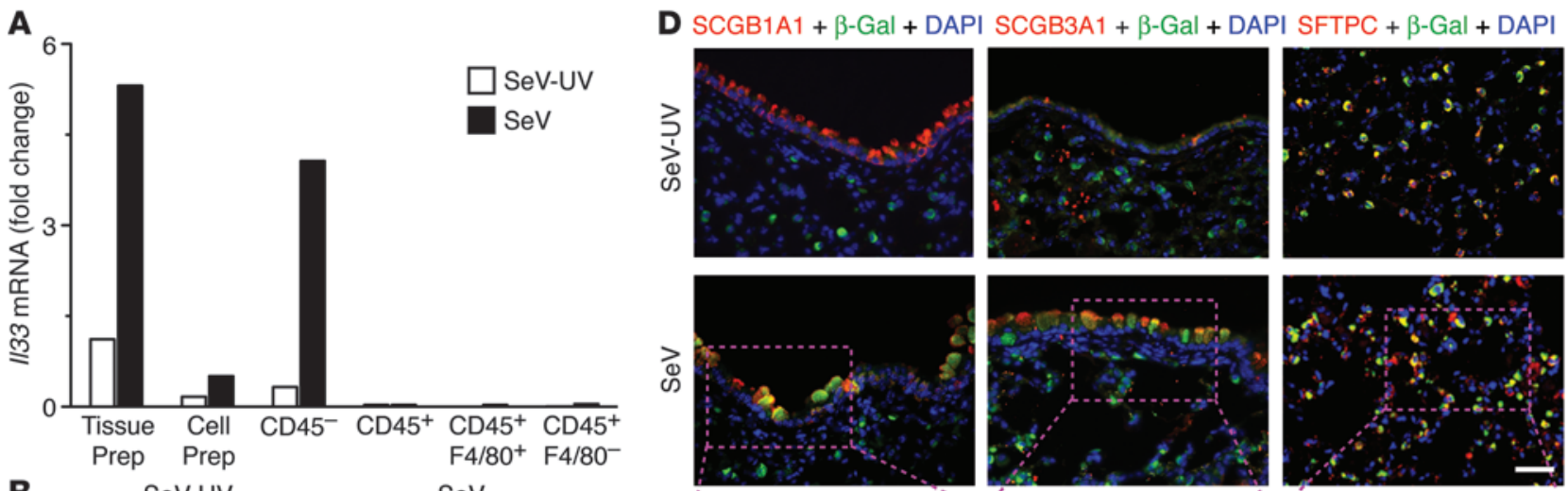

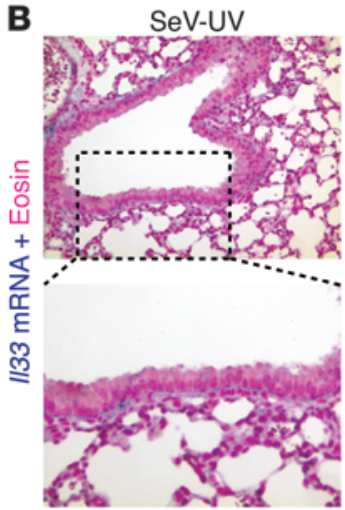

C PAS
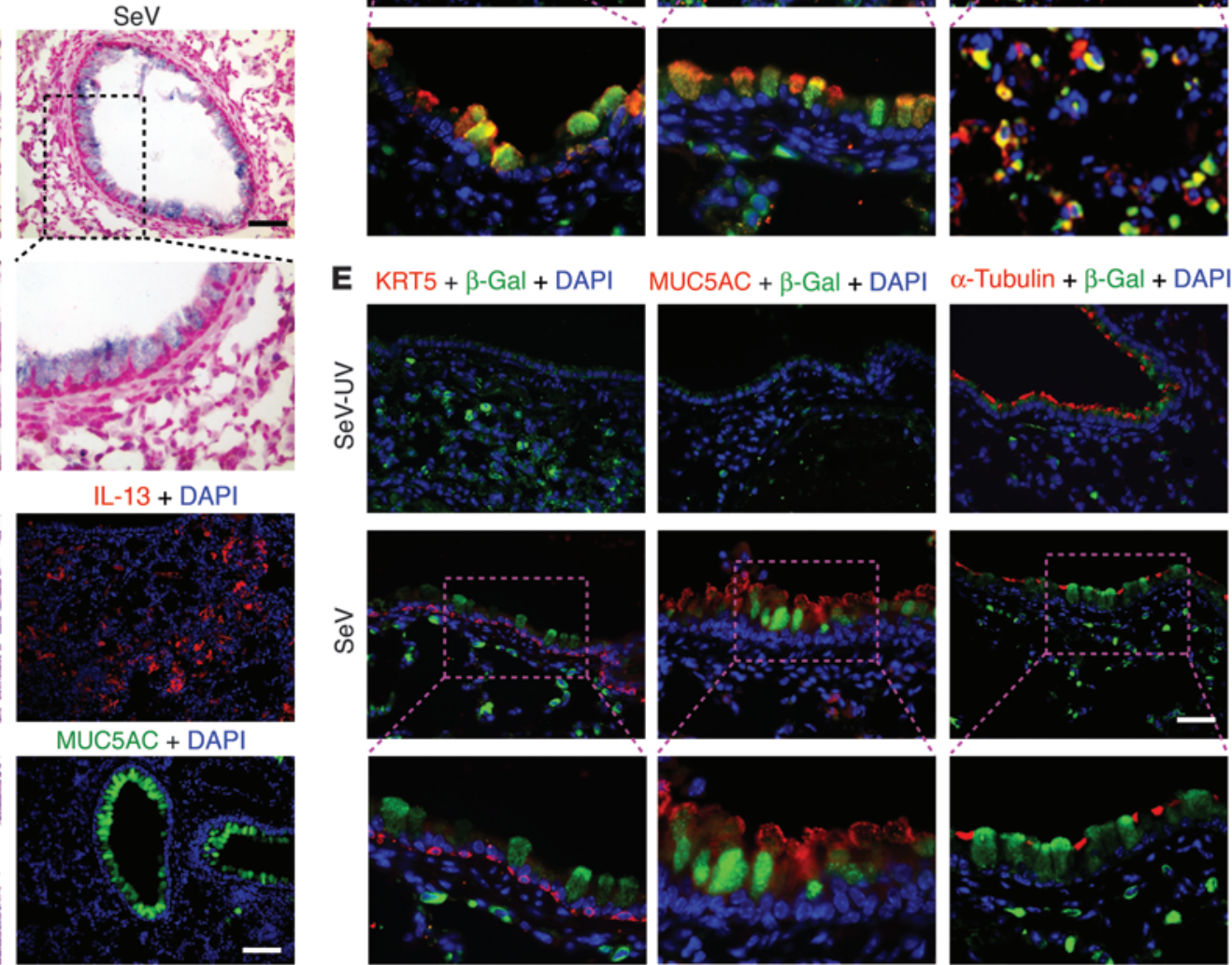

$\alpha-$ Tubulin + $\beta$-Gal + DAPI
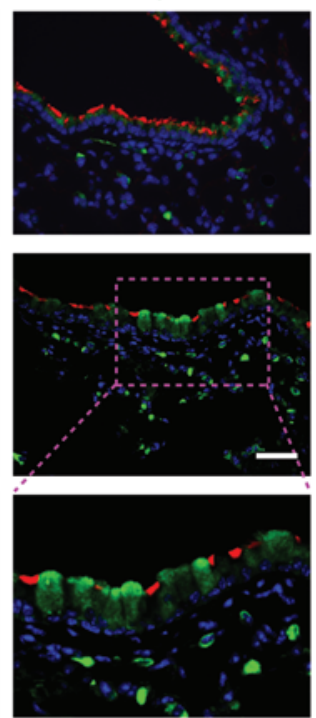

Figure 5

Localization of IL-33 expression to subsets of lung epithelial cells in the postviral mouse model. (A) Levels of /I33 mRNA from lung tissue preparation (Tissue Prep), lung cell preparation before FACS (Cell Prep), and the indicated cell populations purified by FACS from lungs obtained at dpi 49. Values are representative of 3 experiments. (B) Representative photomicrographs showing in situ hybridization for //33 mRNA at dpi 49 . (C) Representative photomicrographs showing PAS staining and IL-13 and MUC5AC immunostaining in I/33Wt/Gt lung sections at dpi 49. (D) Representative photomicrographs showing IL-33 immunostaining (detected with anti- $\beta$-gal antibody) and indicated cell type-specific costaining and DAPI counterstaining in //33 Wt/Gt lung sections at dpi 49. (E) Representative photomicrographs for additional immunostaining using conditions in D. Boxed regions in B-E are shown at higher magnification below (enlarged $\times 2$ ). Scale bars: $200 \mu \mathrm{m}$ (B and C); $100 \mu \mathrm{m}(\mathbf{D}$ and $\mathbf{E})$.

mice (Supplemental Figure 4), thereby excluding the nonspecific staining found for anti-mouse IL-33 antibodies (37). The present approach also offers the advantage of detecting only IL-33 production, not IL-33 bound to its receptor on the target cell. Together, the concerted approaches for IL-33 localization (i.e., PCR assay of FACSpurified cells, in situ hybridization, and IL-33 reporter mice) indicated that IL-33 expression in postviral mice with chronic obstructive lung disease is traceable to a newly induced subset of airway serous cells marked especially by SCGB3A1 expression as well as a constitutive subset of alveolar type 2 cells marked by SFTPC expres- sion. Thus, IL-33 expression marks an airway serous cell population linked previously to repair, renewal, and remodeling in the distal airway epithelium $(34,35,43,44)$ and an alveolar type 2 cell population responsible for the same progenitor functions in the alveolar compartment $(45,46)$. The findings suggested that an IL-33-expressing progenitor cell population might be a feature of chronic obstructive lung disease, so we pursued this possibility in humans.

IL-33-expressing basal cells in COPD in vivo. To translate our findings from the mouse model to humans, we used whole lung explants from recipients undergoing lung transplantation for very severe 

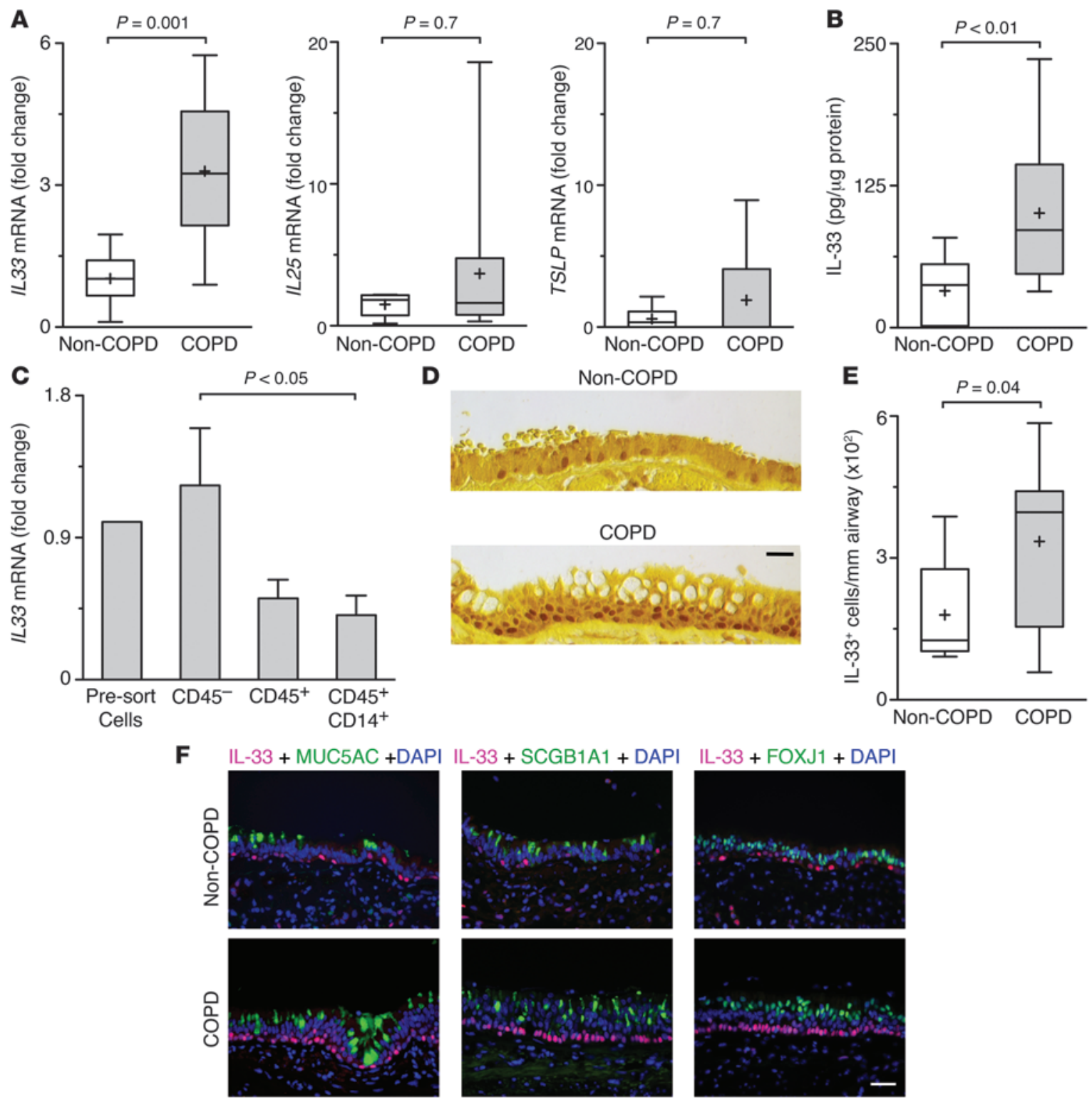

\section{Figure 6}

Selective increase in IL-33-expressing airway epithelial cells in COPD. (A). Levels of IL33, IL25, and TSLP mRNA in non-COPD ( $n=7$ ) and COPD ( $n=15)$ lung tissues. (B) Levels of IL-33, determined by ELISA, using non-COPD ( $n=16$ samples from 9 subjects) versus COPD ( $n=17$ samples from 11 subjects) lung lysates. (C) Levels of IL33 mRNA in lung cells before FACS (Pre-sort Cells) and the indicated cell populations purified by FACS from COPD subjects $(n=4)$ using labeled anti-CD45 and anti-CD14 mAb. (D) Representative photomicrographs of sections of airway epithelium immunostained for IL-33 with DAB (brown) reporter and counterstained with tartrazine (yellow). (E) Number of IL-33+ cell nuclei per millimeter basement membrane in non-COPD $(n=6)$ versus COPD $(n=10)$ lung tissues. (F) Representative photomicrographs of non-COPD and COPD airway epithelium sections immunostained for IL-33 with red (Alexa Fluor 594) reporter; costained for MUC5AC, SCGB1A1, or FOXJ1 with green (Alexa Fluor 488) reporter; and counterstained with DAPI (blue). (A, B, and E) Box represents 25th-75th percentile; line represents median; "+" represents mean; whiskers represent range. (C) Bars represent mean \pm SEM. $P$ values were derived from unpaired Student's $t$ test, but comparisons of medians were also made using Mann-Whitney $U$ test with similar results. Scale bars: $100 \mu \mathrm{m}$.

(GOLD Stage IV) COPD (Supplemental Table 2), compared with lungs from non-COPD donors, to assess expression of TSLP, IL-25, and IL-33. We found significantly increased IL33 mRNA levels in lung samples from COPD versus non-COPD subjects (Figure 6A). To better address the relationship of increased IL-33 expression to airway disease (particularly mucus production), we also examined lung samples directed to airways based on CT imaging (47). In these airway-rich sections, we again found a significant increase in IL33 mRNA in COPD versus non-COPD subjects as well as increased levels of IL13, IL13RA1, and MUC5AC mRNA (Supplemental Figure 5), consistent with our previous observations in smaller groups of subjects $(11,13)$. Moreover, IL33 mRNA levels correlated with MUC5AC and IL13RA1 mRNA levels across the group of COPD and non-COPD lung samples (Supplemental Figure 5). We did not find 
A
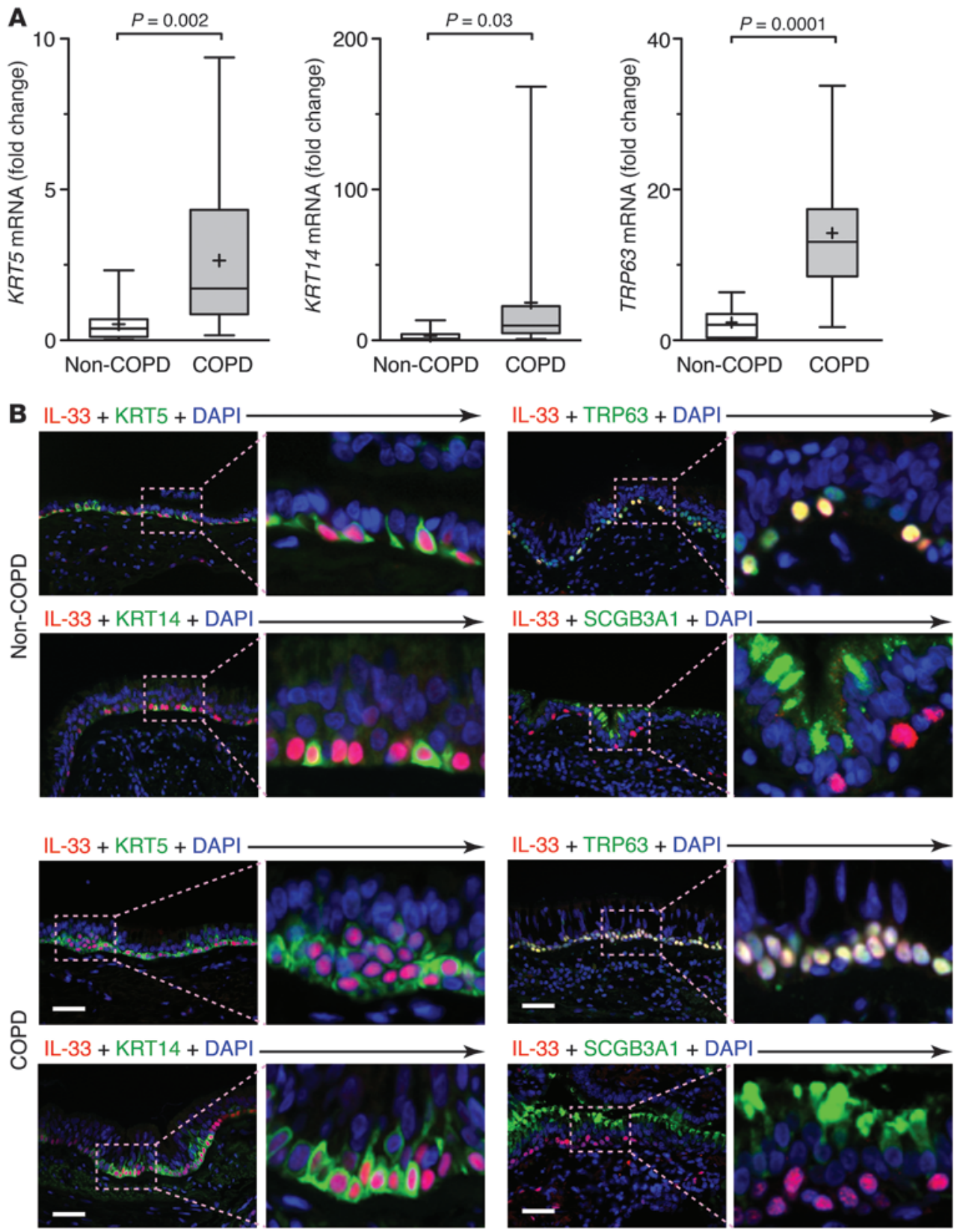

IL-33 + SCGB3A1 + DAPI

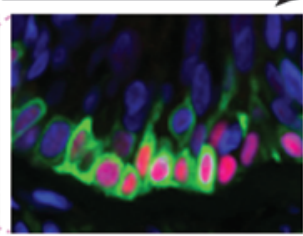

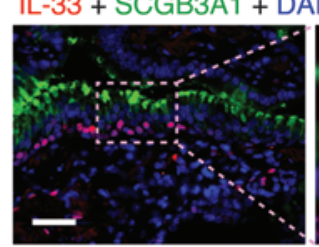

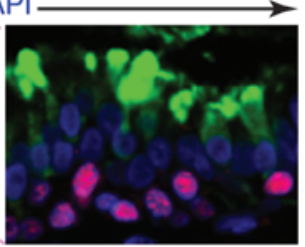

\section{Figure 7}

Localization of IL-33 expression to a subset of airway basal cells in COPD. (A) Levels of KRT5, $K R T 14$, and TRP63 mRNA in non-COPD $(n=18)$ and COPD $(n=21)$ lungs. (B) Representative photomicrographs of nonCOPD and COPD airway epithelium sections immunostained for IL-33; costained for KRT5, KRT14, TRP63, or SCGB3A1; and counterstained with DAPI. Boxed regions are shown at higher magnification at right (enlarged $\times 4)$. Scale bars: $100 \mu \mathrm{m}$. a correlation of IL13 with expression of IL33, IL13RA1, or MUC5AC, perhaps a reflection of the relatively transient and low-level production of cytokines (such as IL-13) versus more stable and higher-level expression of trigger, receptor, and responder components (such as IL-33, IL-13 receptor, and MUC5AC) of the cytokine signaling pathway. Although there was some degree of heterogeneity among individual patients, we found no significant difference in either IL25 or TSLP mRNA levels in COPD versus non-COPD subjects (Figure 6A). The increase in lung IL33 mRNA levels was accompanied by a significant increase in IL-33 protein levels in lung samples from COPD versus non-COPD subjects (Figure 6B).

We next traced the cellular source of increased IL-33 production in the lungs of COPD subjects. Analysis of FACS-purified lung cell populations indicated that the primary source of IL33 mRNA production in COPD was CD45- nonhematopoietic cells (Figure 6C and Supplemental Figure 6). In concert with this finding, IL-33 immunostaining was most intense in airway basal cells present in regions of epithelial hyperplasia and mucous cell remodeling with and without associated bronchial-associated lymphoid tissue (BALT) (Figure 6D and Supplemental Figure 6) and was characterized by an increased number of IL- $33^{+}$airway epithelial cells in samples from COPD versus non-COPD subjects (Figure 6E). These findings were consistent with detection of increased IL33 mRNA in airway-containing samples from COPD versus non-COPD subjects. We found no detectable IL-33 staining in more differentiated airway epithelial cells, i.e., MUC5 $\mathrm{AC}^{+}$mucous cells, SCGB1A1 ${ }^{+}$serous cells, or FOXJ1+ ciliated cells, in non-COPD or COPD subjects (Figure 6F). We did detect IL-33 immunostaining of endothelial cells of the lung microvasculature along with scattered cells in the interstitium, which in both cases was again localized to the nucleus. 
A
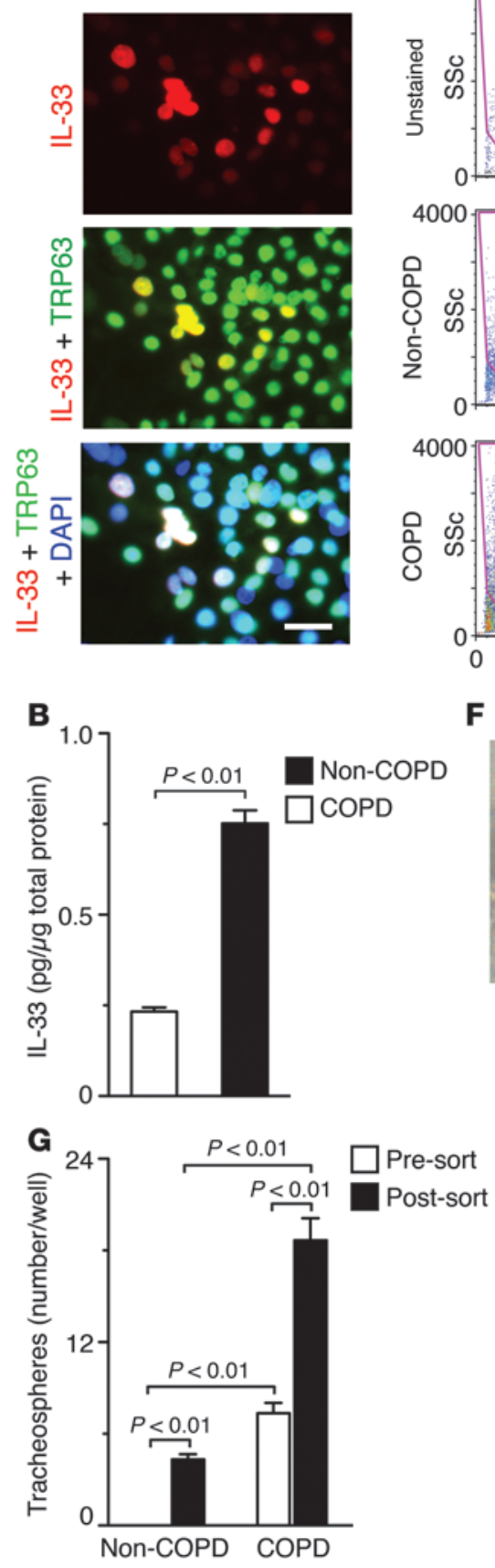
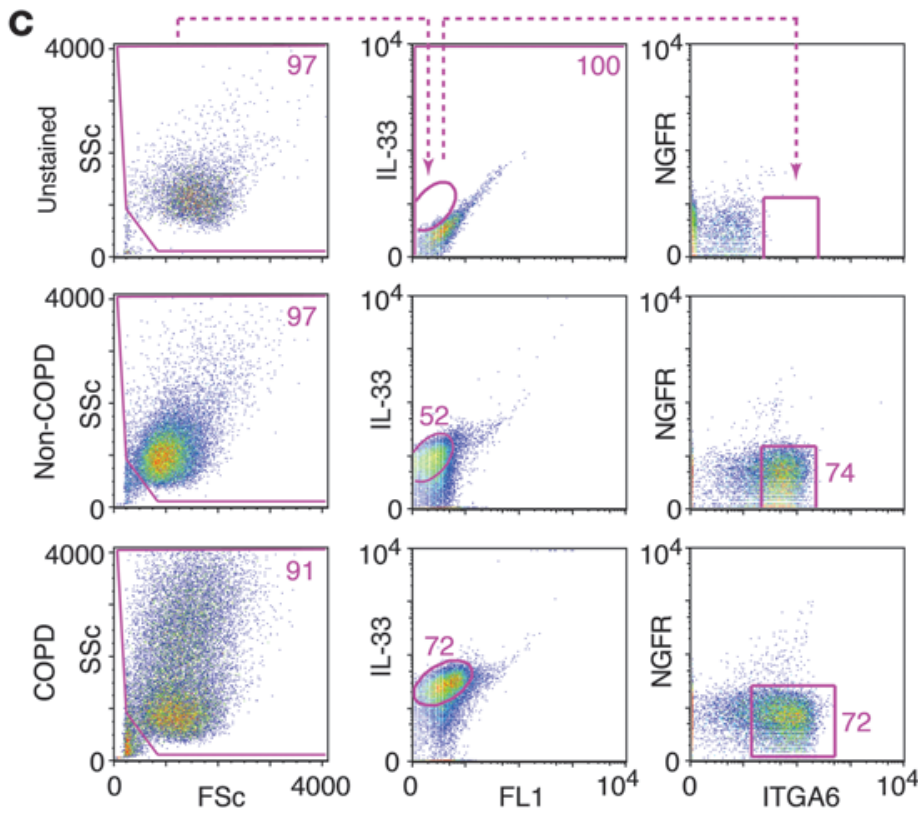

F Phase Contrast

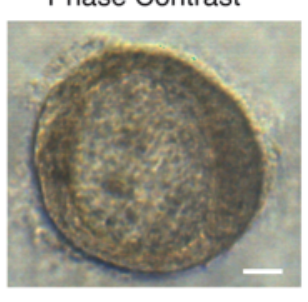

KRT14 + KRT8 + DAPI

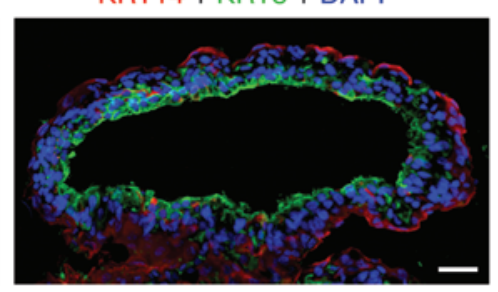

D

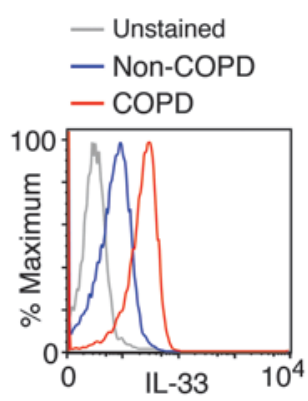

E
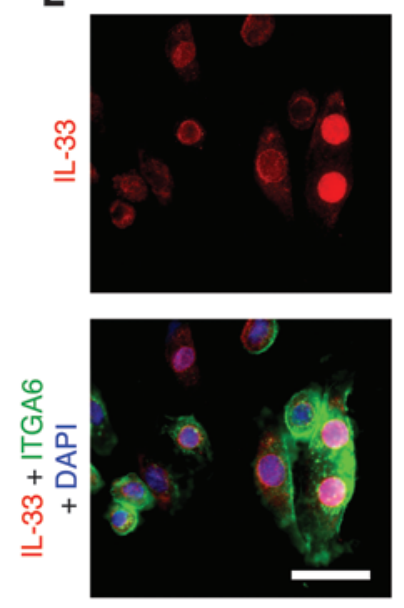

H

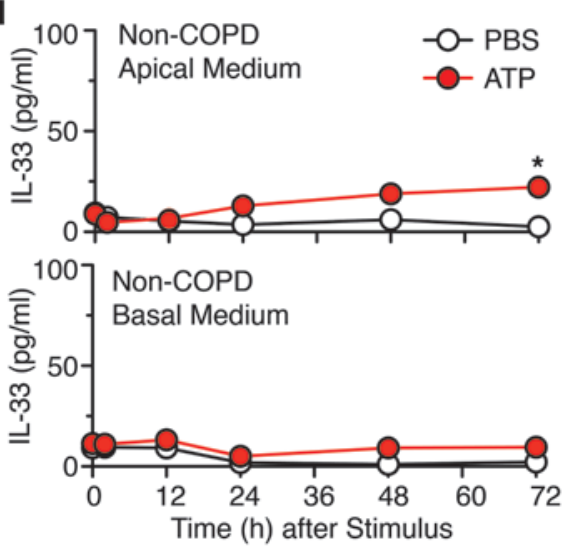

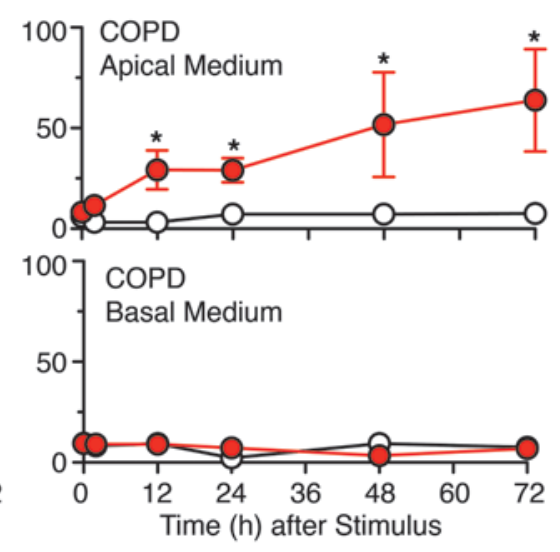

\section{Figure 8}

Basal cell capacity for tracheobronchosphere formation and IL-33 release in COPD. (A) Representative photomicrographs of hTECs isolated from COPD subjects, cultured in 2D submerged conditions for 5 days, and immunostained for IL-33 and TRP63 and counterstained with DAPI. (B) IL-33 levels in cell lysates for non-COPD and COPD subjects ( $n=3$ per group) as in A. (C and D) Flow cytometric analysis of $h$ TECs cultured from COPD and non-COPD subjects ( $n=3$ per group) and then permeabilized and stained for IL-33, ITGA6, and NGFR along with unstained non-COPD control. (C) Representative cytograms. FL1, autofluorescence signal; FSc, forward scatter. Values indicate percent of cells within the designated gate. (D) Histograms. (E) Representative photomicrographs showing IL-33 and ITGA6 immunostaining as in A. (F) Representative photomicrographs of tracheobronchospheres in 3D culture using phase contrast and fluorescent microscopy for immunostaining for ablumenal/ basal KRT14 and lumenal/apical KRT8. (G) Quantitation of tracheobronchosphere formation for basal cells with and without FACS purification from non-COPD $(n=3)$ and COPD $(n=5)$ subjects, using sorting and culture conditions as in C and F. (H) Levels of IL-33 released from hTECs isolated from a COPD and a non-COPD subject, cultured submerged for 5 days, and then incubated with ATP for the indicated time periods. Results are representative of $3-5$ subjects. ${ }^{*} P<0.05$ versus PBS at $0 \mathrm{~h}, 2$-way ANOVA. Scale bars: $25 \mu \mathrm{m}(\mathbf{A}$ and $\mathbf{E}) ; 100 \mu \mathrm{m}(\mathbf{F})$. 


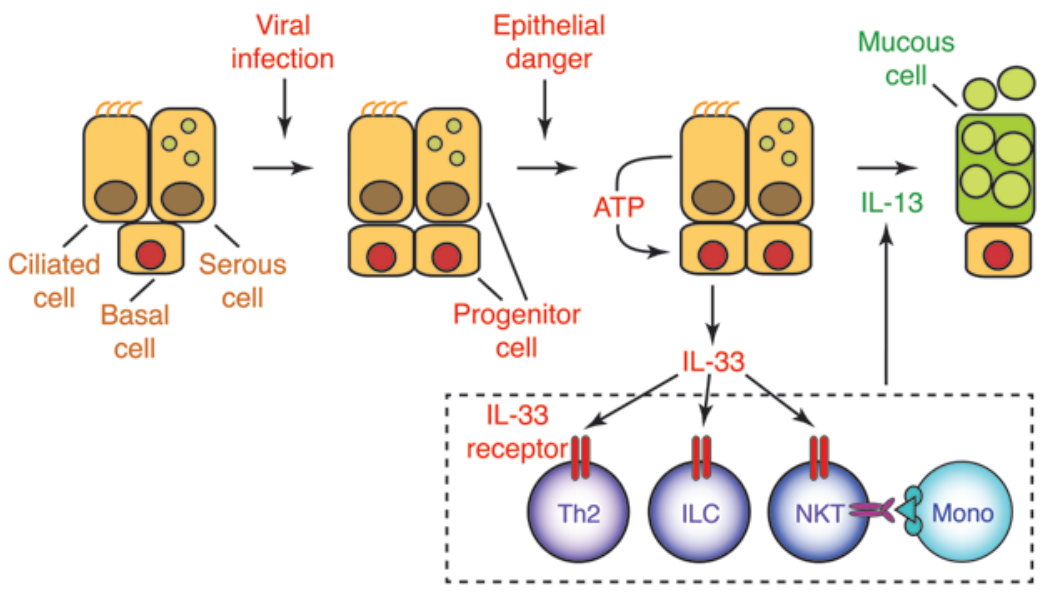

\begin{abstract}
Figure 9
IL-33/IL-13 immune axis in chronic obstructive lung disease. Respiratory viral infection leads to an increase in lung epithelial progenitor cells (airway basal cells in humans, and perhaps airway serous cells and alveolar type 2 cells in mice) that are programmed for increased IL-33 expression. Subsequent epithelial danger signals stimulate ATP-regulated release of IL-33 that acts on immune cells in the lung, e.g., CD4+ Th2 cells, innate lymphoid cells (ILC), and semi-invariant NKT cells with interacting monocytes and macrophages (Mono) to stimulate IL-13 production and consequent airway mucous cell and mucus formation.
\end{abstract}

The increase in IL-33-expressing airway basal cells was accompanied by significant increases in expression of conventional basal cell genes (i.e., KRT5, KRT14, and TRP63) in the lungs of COPD versus non-COPD subjects (Figure 7A). Moreover, IL-33 expression was localized to a subset of $\mathrm{KRT}^{+}, \mathrm{KRT} 14^{+}$, and $\mathrm{TRP} 63^{+}$airway basal cells, again particularly prominent in areas of epithelial hyperplasia and mucous cell remodeling in COPD (Figure 7B). IL-33 did not overlap completely with KRT5, KRT13, or TRP63 immunostaining, which suggests that IL-33-expressing cells represent a distinct airway basal cell niche. We did not detect IL-33 immunostaining in SCGB3A1 ${ }^{+}$airway serous cells (in contrast to the mouse model) or in alveolar or tissue macrophages (Figure 7B and data not shown). Based on the role of TRP63 in suppression of apoptosis and prolongation of epithelial cell survival (48), similar to the case for EpCAM (40), our findings again suggested that IL-33 expression may mark a progenitor cell population.

IL-33-expressing basal progenitor cells in culture. Given the tools available for analysis of lung progenitor cells, we next examined whether the IL-33 expression program continues ex vivo in basal cells isolated from COPD and non-COPD subjects. We used whole lung explants for blunt dissection of airways and then isolation and culture of human tracheobronchial epithelial cells (hTECs) under submerged conditions. These conditions prevented epithelial cell differentiation and thereby preserved airway basal cells, including the IL- $33^{+} \mathrm{TRP} 63^{+}$subset (Figure $8 \mathrm{~A}$ ). We found that, in contrast to submerged culture conditions, IL33 mRNA was 150 fold decreased and IL-33 protein was undetectable with cell differentiation in air-liquid interface conditions (data not shown). Under submerged culture conditions, we also found that levels of IL-33 expression were significantly increased in basal cells from COPD versus non-COPD subjects even after 5 days in culture (Figure 8B). We then subjected hTECs from submerged cultures to intracellular staining for IL-33 along with detection of cell surface markers that are selective for airway basal cells (i.e., nerve growth factor receptor [NGFR] and integrin $\alpha 6$ [ITGA6]) (36). This strategy revealed an association of IL-33 expression with an ITGA $6{ }^{\text {hi NGFR }}{ }^{\text {lo }}$ subpopulation of basal cells in both COPD and non-COPD subjects (Figure 8C), again with increased levels of IL-33 expression in COPD versus non-COPD cells (Figure 8D).

Given the conventional role of basal cells as epithelial progenitors in human airways $(36,42)$ as well as the association of IL-33 expression with typically progenitor cell populations in the mouse model, we next checked for progenitor potential in basal cells isolated from
COPD and non-COPD subjects. We found that IL-33+ basal cells placed into an enriched 3D Matrigel culture system were fully capable of forming structures with fidelity to original airway morphology (designated tracheobronchospheres). After culture of these tracheobronchospheres under differentiation-promoting conditions, phase-contrast microscopy and video microscopy revealed proper ciliary formation and movement (Figure 8F and Supplemental Video 1), and immunofluorescence microscopy showed ablumenal KRT14 and lumenal KRT8 expression, as expected for differentiated airway structure (36). Moreover, we observed a significantly increased efficiency of sphere formation from cultures that were enriched for IL-33-expressing (ITGA6 ${ }^{\text {hiNGFR }}{ }^{\text {lo }}$ ) cells (Figure 8G). Increased sphere formation was also found for cells obtained from COPD versus non-COPD subjects (Figure 8G). These results provide evidence of a progenitor subset of airway basal cells that is marked by IL-33 expression and is increased in COPD.

Our results from the postviral mouse model also indicated that IL-33 must reach the extracellular space and bind to its receptor for the development of lung disease (since disease was blocked using anti-IL1RL1 $\mathrm{mAb}$ and by loss of IL1RL1). We therefore examined the capacity for IL-33 release from basal cells, in light of previous observations that extracellular ATP may act as a danger signal to trigger IL-33 release by activation of purinergic receptor $\mathrm{P} 2 \mathrm{Y}_{2} \mathrm{R}$ signaling (49). Consistent with this concept, direct administration of ATP caused a significant increase in IL-33 release in basal cells cultured from both COPD and non-COPD subjects (Figure $8 \mathrm{H}$ ). Under these conditions, IL-33 was detected exclusively in the apical medium and was found to accumulate at increasing levels over 1-3 days. We found no significant difference in cell morphology or LDH release in ATP- versus PBS-treated COPD or non-COPD subjects (Supplemental Figure 7), consistent with a previous report of noncytotoxic release of IL-33 (49). These data implicate a regulated secretory process by which IL-33 release is directed under conditions of cellular stress. Taken together, these findings indicate that a subset of the airway basal cells is programmed for IL-33 expression and release and that an increase in this cell population can thereby serve as a renewable source of increased IL-33, with the possible downstream consequence of IL-13-dependent lung disease.

\section{Discussion}

It has been difficult to understand how an acute infection might lead to a chronic inflammatory disease. We gained substantial insight into this process with studies of viral infection in mice 
aimed at modeling the initiation, exacerbation, and progression of chronic obstructive lung diseases such as COPD. In our mouse model of this disease process, we uncovered an innate immune axis involving semi-invariant NKT cells and M2 monocytes and macrophages that drives IL-13 production and consequent airway mucus production and hyperreactivity that are characteristic of chronic obstructive lung disease in humans $(10-12,44,50)$. We recognized, however, that there must be upstream factors that more directly link acute viral infection with chronic activation of immune effector cells. In the present study, we assessed the factors best known to regulate IL-13 production, i.e., IL-25, TSLP, and IL-33, and showed that IL-33 was a primary influence on the innate immune axis that drives IL-13-dependent lung disease. Our present findings suggested a primary role for IL-33 in experimental (mouse) and clinical (human) forms of chronic lung disease. (a) In the postviral mouse model, IL-33 expression was selectively induced relative to IL-25 and TSLP, and IL-33/IL-33 receptor signaling was required for chronic increases in IL-13 and airway mucus production. (b) In this model, IL-33 expression was particularly localized to airway serous cells and alveolar type 2 cells, which suggests that a progenitor population might account for long-term IL-33 expression and consequent IL-13-dependent disease. (c) In humans with very severe COPD, IL-33 expression was also selectively increased in lung tissue and was significantly associated with an IL-13 and mucin gene signature, in concert with excess airway mucus production. (d) In these subjects as well as in control subjects, IL-33 expression was particularly localized to nuclei in a subset of airway basal cells that were increased in COPD and are also conventionally linked to progenitor function. (e) In an ex vivo analysis of this basal cell subset, increased nuclear expression of IL-33 was preserved in basal cells cultured from COPD subjects and marked a cell population with increased pluripotency and ATP-regulated release of IL-33. Together, these results suggest a new scheme for an IL-33/ IL-13 immune axis in which an epithelial progenitor cell population can store and release IL-33 to drive downstream IL-13 and consequent airway mucus production that is typical of COPD and perhaps other IL-13-dependent diseases (Figure 9).

An intriguing aspect of our findings is the potential role of an inducible progenitor population in the development of chronic inflammatory disease. Full development of this concept is limited by our present understanding of the progenitor populations in lung injury and repair, including the renewal process that occurs after viral infection. In other mouse models of this process (with and without cell lineage tracking), airway serous cells in the distal airway and alveolar type 2 cells in the alveolar space are generally regarded as progenitors $(34,35,45,46)$, but whether they can be reprogrammed (e.g., by viral infection) to drive chronic disease represents a new paradigm for pathogenesis. Airway and parenchymal basal cells (marked by KRT5 expression) may develop after IAV and $\mathrm{SeV}$ infection (ref. 42 and the present study), but specific diseaseproducing functions of this cell population remain uncertain. For example, we did not find IL-33 expression in this basal cell population in the postviral mouse model of chronic obstructive lung disease. Conversely, it is also generally accepted that airway basal cells are linked to progenitor function in human lungs $(36,41)$; here, we confirmed this proposal and established a further connection to regulated IL-33 expression and release in human lung disease. Since IL-33 expression did not fully coincide with that of other basal cell markers, the IL-33-expressing basal cells (as well as the IL- $33^{+}$serous cells in mice) appear to represent a special- ized airway cell niche. The basis for how such a population might develop still needs to be defined, but our present results suggest that an epigenetic change in this population, perhaps a result of viral reprogramming, might allow for increased abundance of a progenitor subset marked by IL-33 expression.

In pursuing this question, it may be useful to recognize that the progenitor cell program can be relatively inefficient when assessed by tracheobronchosphere formation (e.g., 18 spheres per 3,000 cells seeded, or approximately $0.6 \%$ in samples from COPD subjects), as reported previously $(36,51,52)$. However, sphere-forming efficiency is likely decreased by several of the present experimental conditions, e.g., cellular stress during isolation, cell seeding at low density, and lack of additional supplementation with growth factors and/or fibroblast feeder layers. Further work will be needed to determine the precise relationship of progenitor cell function to IL-33 expression and whether IL-33 serves as a marker or a driver of self renewal and/or mucous cell differentiation. Nonetheless, the increase in sphere-forming efficiency in IL-33-enriched populations in samples from COPD and non-COPD subjects suggests at least some degree of linkage between IL33 gene expression and progenitor cell function.

The progenitor nature of an IL-33-expressing, ATP-responsive cell population could explain the acquired susceptibility to excess mucus production typical of airway disease, but this response may also provide an advantage to the host. Others previously reported a protective role for IL-33 signaling after high-dose intracerebral lymphocytic choriomeningitis virus (LCMV) infection, but found no change in viral clearance at lower inoculum (53), similar to our present results. However, as noted above, it may be useful to consider the chronic condition: long-term IL-33 production may provide for increased airway mucous cells that may be less susceptible to viral infection (54) and facilitate the immune response (55). Similarly, mucus production may facilitate export of noxious materials via cough and/or mucociliary clearance. It is possible that these mucous cell benefits are lost if other environmental stimuli, such as tobacco smoke or allergen, are introduced in conjunction with viral infection to drive excessive mucus production. This issue will require further studies of viral infection in combination with other risk factors for chronic obstructive lung disease.

Our present findings also reinforce and expand the unusual role of IL-33 as a secreted cytokine despite nuclear expression. In the postviral mouse model, extracellular IL-33 acts in a paracrine manner by interacting with its cognate receptor to drive IL-13 production and consequent M2 differentiation and mucus overproduction. In this case, administration of anti-IL1RL1 or engineered deficiency of IL1RL1 blocks these effects $(56,57)$. In our studies of human lung cells, available tools permitted localization of IL-33 to the nuclei of airway basal cells in vivo and in vitro. This situation was consistent with the proposal that IL-33 is constitutively expressed at a high level in basal cells of other epithelial barriers (e.g., skin and genitourinary tract) and can be released with cellular stress $(37,49)$. Whether release of IL-33 for activation of its receptor is regulated by cell turnover or by alarm signals from external stimuli likely depends on the stimulation conditions, but at least in the mouse model, long-term induction and action of IL-33 appears to continue without any obvious stimulus for cell death or damage. Nonetheless, our results using basal cells indicated that further cellular stress and consequent ATP release could stimulate even higher levels of IL-13 expression, M2 differentiation, and mucus production. These characteristics of IL-33 processing and 
action would fit with our observations that it was a more robust marker of chronic lung disease, even at baseline, compared with its downstream readouts.

Our results also help to clarify the cellular target of IL-33 action. Others have proposed a role for IL-33 derived primarily from alveolar macrophages in the development of airway hyperreactivity after IAV infection (24). This conclusion was based on immunostaining with anti-mouse IL-33 antibody (24), which has been problematic, as noted above and in previous reports (37). In contrast, our work and that of by others showed that IL-33 expression was often localized to nonhematopoietic cells $(37,53,58,59$, and the present study). Indeed, our findings fit best with immune cells being a target, rather than a source, of IL-33. The precise and quantitative identification of the immune cell populations that respond to IL-33 with production of IL-13 during postviral disease in mice or chronic obstructive lung disease in humans is still being fully defined. Our initial study of this issue pointed to a predominant functional role for NKT cells and monocytes/macrophages (11), but we also recognized that IL-13-producing innate lymphoid cells were also activated in the postviral mouse model $(60,61)$. A comprehensive analysis of IL-13-producing cells in the mouse model and in human subjects - and, ultimately, selective blockade of these populations - will be required to fully define this issue. It remains possible that IL-33 has additional effects on airway epithelial (and endothelial) cells in the lung, as described previously $(62,63)$, but the functional consequences of this action under healthy or disease conditions remain uncertain.

Others have also proposed a role for IL-25 and TSLP in the development of chronic obstructive lung disease due to asthma $(26,27)$, and, to a much more limited extent, in COPD (25). We did not detect significant differences in lung levels of IL25 or TSLP mRNA in our cohort of COPD patients with very severe disease compared with non-COPD controls, but we cannot exclude a role for these cytokines in controlling IL-13 production or some other aspect of lung disease. In fact, we did observe upregulation of IL-25 and TSLP expression in the lungs of some subjects with severe COPD, but not all. Given the potent action of IL- 25 on induction of IL-13 expression, at least in mice, it is possible that IL-25 (with or without synergy with IL-33) could contribute to overproduction of mucus in some individuals. In the case of TSLP, however, we found only low levels of expression in COPD and a decrease in expression after viral infection in mice. While further studies of TSLP function need to be done, our present data suggest that the contributions of TSLP to obstructive lung disease may not derive from its direct actions in the lung. TSLP is reported to have potent effects in the skin that may lead to allergic asthma (64), but there is no connection as yet to nonallergic lung disease.

Similarly, population studies indicate an association of SNPs near ILIRL1 on chromosome 2 and IL33 on chromosome 9 with the development of asthma (65-67), but to our knowledge, no similar associations have been reported for COPD thus far (68). Moreover, the present results suggest that environmental agents (i.e., viral infection) may alter IL-33 expression, making it likely that epigenetic approaches may be more informative than studies of gene sequence in identifying a basis for COPD-related differences in airway epithelial cells. Similarly, we do not yet understand the genetic or acquired regulatory events that control IL33 gene expression or IL-13 protein processing in progenitor cells. There is a report of Notch dependency for IL-33 expression in vascular endothelial cells (69), but whether this system is involved in IL-33 expression in airway epithelial cells or has any role in inflammatory lung disease still needs to be defined.

In summary, we used a mouse model of chronic obstructive lung disease to guide our understanding of the immune pathways that contribute to similar disease due to COPD in humans. In both the mouse model and in COPD subjects, we detected induction of an IL-33-expressing epithelial cell population with progenitor characteristics that could account for long-term activation of an innate IL-13 immune axis. The consequent accumulation of inflammatory airway mucus is critical to the morbidity and mortality of chronic lower respiratory disease $(31,32,70)$, which now ranks as the third most common cause of death in the United States and the fifth worldwide $(71,72)$. The relationship of the epithelial cell IL-33-to-immune cell IL-13 pathway with other risk factors for COPD, such as tobacco smoking, still needs to be defined. Nonetheless, our results suggest that viral infection is a suitable candidate to drive acute exacerbations with long-term consequences for progression and consolidation of chronic obstructive lung disease. In fact, viral infection appears significantly more potent than smoke exposure as a stimulus of airway inflammatory disease under experimental conditions $(73,74)$. This issue will be better settled as key immune components, e.g., IL-33 and IL-13, are effectively blocked in patients with chronic lung disease. The present findings lay the groundwork for this therapeutic strategy and provide a rational basis for how acute infectious agents (such as common respiratory viruses) can reprogram long-term progenitor cells (such as airway basal cells) to cause chronic inflammatory disease.

\section{Methods}

Mouse generation and treatment. Male mice (6-12 weeks of age) on a C57BL/6 background were used for all experiments. WT C57BL/6J mice were obtained from Jackson Laboratories. Il25//- mice (75) were provided by A. McKenzie (Cambridge, England) and backcrossed to C57BL/6 for a total of 10 generations. Ill17 rb- mice on a C57BL/6 background as well as antimouse IL-25 (clone M819) and IL17RB (clone M735) mAbs (76) were provided by A. Budelsky (Amgen, Seattle, Washington, USA). Illrl1 ${ }^{-/}$mice $(56,57)$ were obtained from P. Bryce (Northwestern University, Chicago, Illinois, USA). $I l 3^{G t / G t}$ mice were generated with insertion of a gene trap cassette containing the LacZ (bgeo) reporter into intron 1 of the Il33 locus, as described previously (37). Anti-mouse IL1RL1 (clone M955) and control $\mathrm{IgG}_{1}$ (clone 4D2) mAbs were provided by D. Smith (Amgen, Seattle, Washington, USA). Mice were inoculated with either $\operatorname{SeV}\left(1 \times 10^{5} \mathrm{pfu}\right)$, an equivalent amount of SeV-UV, or PBS, as described previously (11). Inoculation with SeV-UV and PBS gave identical results and were therefore treated as equivalent control conditions. For mAb blocking experiments, mice were injected at weekly intervals beginning at dpi 12 with anti-IL-25 mAb

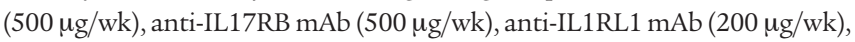
or an equivalent amount of control Ig given intraperitoneally, as described previously $(76,77)$. For cytokine administration, recombinant mouse IL-25 and IL-33 (R\&D Systems) were reconstituted per the manufacturer's recommendations, diluted in PBS, and administered intranasally.

Gene expression microarray. Gene expression analysis was performed using Illumina Mouse-WG6 v2 BeadChips (Illumina). Total RNA was isolated from lung homogenates using the RNEasy kit (Qiagen) and was amplified and biotinylated using the Illumina TotalPrep Kit (Ambion). Treatment and conditions were randomized across BeadChip slides to avoid confounding (78). Hybridization and scanning of BeadChip arrays was performed with BeadStudio 3.0 software (Illumina). Microarray normalization and analysis was performed with Bioconductor packages in the 
$R$ programming environment (79). Raw bead-level intensity data import and background correction was performed using the beadarray package (80). Each Mouse-WG6-v2 BeadChip array consists of 2 strips, which must be treated separately through bead-type summarization due to potential variation in the data from the 2 strips (81). For each strip, low-level analysis and quality control was performed using the beadarray package (82). The BASH algorithm was applied to mask beads affected by large spatial artifacts (83), followed by bead-type summarization, including outlier bead removal (82). Summarized data were $\log _{2}$ transformed, then quantile normalized (84). Bead types whose expression was not detected on any strip in the experiment (detection $P>0.05$ ) were removed. Data from the 2 independently processed strips per array were then merged. Differential expression was assessed using linear models and empirical Bayes moderated $\mathrm{F}$ statistics, as implemented in the LIMMA package (85). To improve interpretation (86), bead types were annotated using annotations from Illumina (87), ReMOAT (88), AILUN (89), AceView (90), and Ensembl (91). Differences in gene expression were considered significant if $P<0.05$ after adjustment for multiple testing (92), limiting the false discovery rate to $<5 \%$. Visualization and plotting was performed using DecisionSite for Functional Genomics (TIBCO Spotfire). Microarray data were deposited in GEO (accession no. GSE49603).

Real-time PCR assay. RNA was purified from lung homogenates using TRIzOL (Invitrogen) and converted to cDNA using a High-Capacity cDNA Archive kit (Applied Biosystems). Target mRNA was quantified by realtime PCR assay using specific fluorogenic probes and primer sets and the Fast Universal PCR Master Mix system (Applied Biosystems). See Supplemental Table 3 for primer-probe sets for human and mouse mRNA targets. All probes were designed to span introns and did not react with genomic DNA. Samples were assayed with the $7300 \mathrm{HT}$ or 7500 Fast Real-Time PCR System and analyzed using Fast System Software (Applied Biosystems). For human tissues, Ct values were normalized to GAPDH mRNA and expressed as fold change relative to the mean $\mathrm{Ct}$ value for the non-COPD control samples using the $\Delta \Delta \mathrm{Ct}$ method (93). For mice, cDNA mRNA copy numbers were corrected for Gapdh mRNA and expressed as fold change relative to the mean expression level in control mice.

Immunohistochemistry. Tissues were fixed with $10 \%$ formalin, embedded in paraffin, cut into $5-\mu \mathrm{m}$ sections, and adhered to charged slides. Sections were deparaffinized in Fisherbrand CitroSolv (Fisher), hydrated, and treated with heat-activated antigen unmasking solution (Vector Laboratories). Immunostaining was performed using primary antibodies (Supplemental Table 4) followed by labeled secondary antibodies. Antibody binding was detected using diaminobenzidine (DAB) with hematoxylin (in mouse) or tartrazine (in human) counterstain for brightfield microscopy, or with tyramide-based signal amplification using Alexa Fluor 488 or Alexa Fluor 594 fluorochromes (Invitrogen) for immunofluorescence microscopy. In mouse lung tissue, the number of $\mathrm{MUC} \mathrm{AC}^{+}$cells was quantified using Image software (NIH). In human tissues, the number of $\mathrm{IL}^{-} 33^{+}$cells was quantified using Nanozoomer (Hamamatsu) virtual microscopy and Viziomorph software (Visiopharm).

FACS analysis. For immune cell experiments, single-cell suspensions were generated from minced lung tissue that was subjected to collagenase (Liberase Blendzyme III, Roche), hyaluronidase (Sigma-Aldrich), and DNAse I (grade II, Roche) digestion for 45 minutes at $37^{\circ} \mathrm{C}$. After FcR blockade, lung cell suspensions were incubated with labeled antibodies and sorted using a Moflo (DAKO-Cytomation) high-speed cell sorter. Nonviable cells were excluded from analysis by 7-AAD staining. Mouse cells were immunostained with PE-Cy7-conjugated anti-mouse F4/80 (Ebioscience) and FITC-Cy5.5-conjugated anti-mouse CD45 (Ebioscience), and human cells were immunostained with APC-conjugated anti-human CD14 (BD Biosciences) and biotin-conjugated anti-human CD45 (BD
Biosciences). For hTEC experiments, cells were detached from inserts with trypsin/EDTA solution and immunostained using anti-NGFR rabbit Ab (Abcam) detected with APC-conjugated anti-rabbit Ab (Santa Cruz) and PE-Cy7-conjugated anti-ITGA6 rat Ab (Ebioscience). For IL-33 costaining, cells were then fixed with $4 \%$ paraformaldehyde, permeabilized with $0.5 \%$ IGEPAL (Sigma-Aldrich), immunostained with biotinylated anti-IL-33 $\mathrm{mAb}$ (clone Nessy-1, Enzo Life Biosciences) and PE-labeled streptavidin (BD Pharmingen), and costained using anti-NGFR rabbit Ab (Abcam) detected with APC-conjugated anti-rabbit Ab (Santa Cruz) and PE-Cy7conjugated anti-ITGA6 rat Ab (Ebioscience). Flow cytometry results were plotted and analyzed using FlowJo software (TreeStar).

In situ bybridization. Tissue sections were pretreated to block nonspecific binding and then hybridized overnight with digoxygenin-labeled antisense RNA probe for Il33 mRNA, as described previously (94). After stringent washing and RNase treatment, Il33 mRNA was detected using an antibodylinked alkaline phosphatase reaction with BM purple chromophore (Roche Diagnostics), and sections were counterstained with nuclear fast red.

Cytokine ELISA. ELISA for human and mouse IL-33 was performed using IL-33 Duosets (R\&D Systems) according to the manufacturer's protocol. For mouse and human sample preparation, lung tissues were homogenized in T-PER reagent containing HALT protease inhibitors (Pierce), and the tissue lysate was diluted ( $2 \mu \mathrm{l}$ in $100 \mu \mathrm{l}$ of $1 \% \mathrm{BSA})$ and incubated on plates for 2 hours at $25^{\circ} \mathrm{C}$. Plates were washed, then incubated with biotinylated detection antibody followed by streptavidin HRP. Antibody binding was detected with TMB substrate (KPL), quenched with $\mathrm{HCl}(1 \mathrm{M})$, and assayed by absorbance at $450 \mathrm{~nm}$. IL-33 level was quantified on a 7-point standard curve and normalized to total protein in the sample. For mouse samples, lung tissues were homogenized in T-PER reagent (Pierce) with the mammalian protease inhibitor cocktail (Sigma-Aldrich), and ELISA was performed as described above.

Human lung samples. Clinical samples were obtained from lung explants harvested at the time of lung transplantation from COPD recipients $(n=35)$ with very severe disease (GOLD Stage IV) during the period from 2005 to 2012 at Barnes-Jewish Hospital (St. Louis, Missouri, USA). Control samples were obtained from non-COPD donors $(n=18)$ using both lung explants that were otherwise not useable for transplantation and excess lung tissue that was resected for downsizing. For the first analysis of gene expression, lung explant samples were obtained from a group of COPD recipients $(n=7)$ and non-COPD donors $(n=15)$ without airway localization. For the second analysis of gene expression, samples were obtained from a group of COPD recipients $(n=16)$ and non-COPD donors $(n=7)$, using CT imaging of the lung explant to ensure inclusion of airway tissue, as described previously (47). Each analysis consisted of samples from COPD recipients with the same clinical characteristics (Supplemental Table 2).

Airway epithelial cell culture. Primary culture hTECs were established from tracheobronchial specimens of COPD recipients and non-COPD donors using 2D submerged and air-liquid interface cultures as well as $3 \mathrm{D}$ sphere culture conditions, as described previously $(36,95)$. For the present experiments, cells were grown in UNC BEGM without retinoic acid (RA) or ALI medium (96) in 12-mm Transwell permeable support plates (Corning Life Sciences). For 2D cultures, cells in submerged format were seeded at 20,000 cells/well, grown to confluence (3-5 days) in BEGM (upper and lower chambers), and used for study. The air-liquid interface condition was established by removing medium from the upper chamber and changing the medium in the lower compartment to UNC ALI medium supplemented with RA (50 nM), then culturing cells for an additional 21 days. For IL-33 release experiments in submerged $2 \mathrm{D}$ cultures, cells were incubated with ATP $(10 \mu \mathrm{M})$ from Cytoskeleton or PBS vehicle, and IL-33 level was determined by ELISA and LDH level by activity assay (Sigma-Aldrich). For 3D tracheobronchosphere cultures estab- 
lished on pre- and post-sort (IL-33-enriched) populations, cells were resuspended in BEGM, mixed 1:1 with growth factor-reduced Matrigel (BD Biosciences), and plated at $3 \times 10^{3}$ cells per $100 \mu \mathrm{l}$ in Transwells. After 7 days, medium in lower chamber was changed from BEGM to UNC ALI supplemented with RA, and spheres were cultured for 30 days. Differentiated spheres were counted and were assessed for ciliary beating using the SAVA system. Spheres were harvested from Matrigel using cell recovery solution (BD Biosciences) and embedded in OCT (Tissue-Tek) for frozen sectioning and immunostaining.

Statistics. Unless otherwise stated, all data are presented as mean \pm SEM, and 2-tailed unpaired Student's $t$ test was used to assess statistical significance between means. In all cases, a $P$ value less than 0.05 was considered significant. All values are representative of at least 3 experiments.

Study approval. All animal experiments were performed using protocols approved by the Washington University Animal Studies Committee. All human studies were conducted with protocols approved by the Washington University Institutional Review Board, and subjects provided informed consent prior to their participation in the study.

1. Tuder RM, Petrache I. Pathogenesis of chronic obstructive pulmonary disease. J Clin Invest. 2012; 122(8):2749-2755

2. Sethi S, Murphy TF. Infection in the pathogenesis and course of chronic obstructive pulmonary disease. NEngl J Med. 2008;359(22):2355-2365.

3. Seemungal T, et al. Respiratory viruses, symptoms, and inflammatory markers in acute exacerbations and stable chronic obstructive pulmonary disease. Am J Respir Crit Care Med. 2001;164(9):1618-1623.

4. Rohde $G$, et al. Respiratory viruses in exacerbations of chronic obstrutive pulmonary disease requiring hospitalisation: a case-control study. Thorax. 2003; 58(1):37-42.

5. Cameron RJ, de Wit D, Welsh TN, Ferguson J, Grissell TV, Rye PJ. Virus infection in exacerbations of chronic obstructive pulmonary disease requiring ventilation. Intensive Care Med. 2006; 32(7):1022-1029.

6. Jenkins CR, et al. Seasonality and determinants of moderate and severe COPD exacerbations in the TORCH study. Eur Respir J. 2012;39(1):38-45.

7. Dimopoulos G, et al. Viral epidemiology of acute exacerbations of chronic obstructive pulmonary disease. Pulm Pharmacol Ther. 2012;25(1):12-18.

8. Mallia P, et al. Experimental rhinovirus infection as a human model of chronic obstructive pulmonary disease exacerbation. Am J Respir Crit Care Med. 2011;183(6):734-742

9. Mallia $\mathrm{P}$, et al. Rhinovirus infection induces degradation of antimicrobial peptides and secondary bacterial infection in COPD. Am J Respir Crit Care Med. 2012;186(11):1117-1124.

10. Walter MJ, Morton JD, Kajiwara N, Agapov E, Holtzman MJ. Viral induction of a chronic asthma phenotype and genetic segregation from the acute response. J Clin Invest. 2002;110(2):165-175.

11. Kim EY, et al. Persistent activation of an innate immune response translates respiratory viral infection into chronic inflammatory lung disease. Nat Med. 2008;14(6):633-640.

12. Agapov E, et al. Macrophage chitinase 1 stratifies chronic obstructive lung disease. Am J Respir Cell Mol Biol. 2009;41(4):379-384.

13. Alevy Y, et al. IL-13-induced airway mucus production is attenuated by MAPK13 inhibition. J Clin Invest. 2012;122(12):4555-4568.

14. Saenz SA, Taylor BC, Artis D. Welcome to the neighborhood: epithelial cell-derived cytokinens license innate and adaptive immune responses at mucosal sites. Immunol Rev. 2010;226:172-190.

15. Liew FY, Pitman NI, McInnes IB. Disease-associated functions of IL-33: the new kid in the IL-1 family. Nat Rev Immunol. 2010;10(2):103-110.

\section{Acknowledgments}

We thank the staff in the Flow Cytometry Core in the Siteman Cancer Center and the Morphology Core in Pulmonary and Critical Care Medicine for outstanding technical support and Pascale Mercier (CNRS, Toulouse, France) for transfer of Il33 gene trap mice. This work was supported by the NIH (NIAID grants AADCRC U19-AI070489 and MRCE U54-AI05160, and NHLBI grants P01-HL29594, R01-HL073159, CADET P50-HL107183, and K08HL083095), ATS/COPD Foundation, Children's Discovery Institute, and the Martin Schaeffer Fund.

Received for publication June 28, 2012, and accepted in revised form June 13, 2013.

Address correspondence to: Michael J. Holtzman, Washington University School of Medicine, Campus Box 8012, 660 South Euclid Avenue, Saint Louis, Missouri 63110, USA. Phone: 314.362.8970; Fax: 314.362.9009; E-mail: holtzmanm@wustl.edu.
16. Zhou B, et al. Thymic stromal lymphopoietin as a key initiator of allergic airway inflammation in mice. Nat Immunol. 2005;6(10):1047-1053.

17. Ballantyne SJ, et al. Blocking IL-25 prevents airway hyperresponsiveness in allergic asthma. J Allergy Clin Immunol. 2007;120(6):1324-1331.

18. Terashima A, et al. A novel subset of mouse NKT cells bearing IL-17 receptor B responds to IL-25 and contributes to airway hyperreactivity. $J$ Exp Med. 2008;205(12):2727-2733.

19. Kearley J, Buckland KF, Mathie SA, Lloyd CM. Resolution of allergic inflammation and airway hyperreactivity is dependent upon disruption of the T1/ST2-IL-33 pathway. Am J Respir Crit Care Med. 2009;179(9):772-781.

20. Liu X, Li M, Wu Y, Zhou Y, Zeng L, Huang T. AntiIL-33 antibody treatment inhibits airway inflammation in a murine model of allergic asthma. Biochem Biophys Res Commun. 2009;386(1):181-185.

21. Wills-Karp M, et al. Trefoil factor 2 rapidly induces interleukin 33 to promote type 2 immunity during allergic asthma and hookworm infection. J Exp Med. 2012;209(3):607-622.

22. Walzl G, et al. Inhibition of T2/ST2 during respiratory syncytial virus infection prevents $\mathrm{T}$ helper cell type 2 (Th2)- but not Th2-driven immunopathology. J Exp Med. 2001;193(7):785-792.

23. Graham BS, Perkins MD, Wright PF, Karzon DT. Primary respiratory syncytial virus infection in mice. J Med Virol. 1988;26(2):153-162.

24. Chang $Y$, et al. Innate lymphoid cells mediate influenza-induced airway hyper-reactivity independently of adaptive immunity. Nat Immunol. 2011; 12(7):631-638.

25 . Ying $S$, et al. Expression and cellular provenance of thymic stromal lymphopoietin and chemokines in patients with severe asthma and chronic obstructive pulmonary disease. J Immunol. 2008; 181(4):2790-2798.

26 . Shikotra A, et al. Increased expression of immunoreactive thymic stromal lymphopoietin in patients with severe asthma. J Allergy Clin Immunol. 2012; 129(1):104-111.

27. Corrigan CJ, et al. T-helper cell type 2 (Th2) memory $\mathrm{T}$ cell-potentiating cytokine IL- 25 has the potential to promote angiongenesis in asthma. Proc Natl Acad Sci U S A. 2011;108(4):1579-1584.

28. Kurowska-Stolarska M, et al. IL-33 amplifies the polarization of alternatively activated macrophages that contribute to airway inflammation. J Immunol. 2009;183(10):6469-6477.

29. Prefontaine D, et al. Increaesed IL-33 expression in epithelial cells in bronchial asthma. J Allergy Clin Immunol. 2010;125(3):752-754.
30. Kuyper LM, et al. Characterization of airway plugging in fatal asthma. Am J Med. 2003;115(1):6-11.

31. Hogg JC, et al. The nature of small-airway obstruction in chronic obstructive pulmonary disease. NEngJ Med. 2004;350(26):2645-2653.

32. Brito-Mutunayagam R, Appleton SL, Wilson DH, Ruffin RE, Adams RJ. Global initiative for chronic obstructive lung disease stage 0 is associated with excess FEV1 decline in a representative population sample. Chest. 2010;138(3):605-613.

33. Kim V, et al. The chronic bronchitic phenotype of COPD. Chest. 2011;140(3):626-633.

34. Hong KU, Reynolds SD, Giangreco A, Hurley CM, Stripp BR. Clara cell secretory protein-expressing cells of the airway neuroepithelial body microenvironment include a label-retaining subset and are critical for epithelial renewal after progenitor cell depletion. Am J Respir Cell Mol Biol. 2001;24(6):671-681.

35. Rawlins EL, et al. The role of Scgb1a1+ Clara cells in the long-term maintenance and repair of lung airway, but not alveolar, epithelium. Cell Stem Cell. 2009;4(6):525-534.

36. Rock JR, et al. Basal cells as stem cells of the mouse trachea and human airway epithelium. Proc Natl Acad SciU S A. 2009;106(31):12771-12775.

37. Pichery M, et al. Endogenous IL-33 is highly expressed in mouse epithelial barrier tissues, lymphoid organs, brain, embryos, and inflamed tissues: in situ analysis using a novel Il-33-LacZ gene trap reporter strain. J Immunol. 2012;188(7):3488-3495.

38. Angkasewinai $P$, et al. Interleukin 25 promotes the initiation of proallergic type 2 responses. J Exp Med. 2007;204(7):1837-1847.

39. Fujino $\mathrm{N}$, et al. A novel method for isolating individual cellular components from the adult human distal lung. Am J Respir Cell Mol Biol. 2012; 46(4):422-430.

40. Karimi-Busheri F, Zadorozhny V, Li T, Shawler DL, Fakhrai H. Pivotal role of CD38 biomarker in combination with CD24, EpCAM, and ALDH for identification of $\mathrm{H} 460$ derived lung cancer stem cells. J Stem Cells. 2011;6(1):9-20.

41. Hong KU, Reynolds SD, Watkins S, Fuchs E, Stripp BR. Basal cells are a multipotent progenitor capable of renewing bronchial epithelium. Am J Pathol. 2004; 164(2):577-588.

42. Kumar PA, et al. Distal airway stem cells yield alveoli in vitro and during lung regeneration following H1N1 influenza infection. Cell. 2011;147(3):525-538.

43. Evans CM, et al. Mucin is produced by Clara cells in the proximal airway of antigen-challenged mice. Am J Respir Cell Mol Biol. 2004;31(4):382-394.

44. Tyner JW, et al. Blocking airway mucous cell metaplasia by inhibiting EGFR antiapoptosis and IL-13 
transdifferentiation signals. J Clin Invest. 2006; 116(2):309-321.

45. Evans MJ, Cabral LJ, Stephens RJ, Freeman G. Renewal of alveolar epithelium in the rat following exposure to NO2. Am J Pathol. 1973;70(2):175-198.

46. Evans MJ, Cabral LJ, Stephens RJ, Freeman G Transformation of alveolar type 2 cells to type 1 cells following exposure to NO2. Exp Mol Pathol. 1975; 22(1):142-150

47. Deslee G, et al. Cigarette smoke induces nucleic acid oxidation in lung fibroblasts. Am J Respir Cell Mol Biol. 2010;43(5):576-584.

48. Rocco JW, Leong CO, Kuperwasser N, DeYoung MP, Ellisen LW. p63 mediates survival in squamous cell carcinoma by suppression of p73-dependent apoptosis. Cancer Cell. 2006;9(1):45-56.

49. Kouzaki H, Iijima K, Kobayashi T, O’Grady SM, Kita $\mathrm{H}$. The danger signal, extracellular ATP, is a sensor for an airborne allergen and triggers IL-33 release and innate Th2-type responses. J Immunol. 2011; 186(7):4375-4387.

50. Holtzman MJ. Asthma as a chronic disease of the innate and adaptive immune systems responding to viruses and allergens. J Clin Invest. 2012; 122(8):2741-2748.

51. Teisanu RM, et al. Functional analysis of two distinct bronchiolar progenitors during lung injury and repair. Am J Respir Cell Mol Biol. 2010;44(6):794-803.

52. Hegab AE, et al. Novel stem/progenitor cell population from murine tracheal submucosal gland ducts with multipotent regenerative potential. Stem Cells. 2011;29(8):1283-1293

53. Bonilla WV, et al. The alarmin interleukin-33 drives protective antiviral CD8+ T cell responses. Science. 2012;335(6071):984-989.

54. Villenave R, et al. Cytopathogenesis of Sendai virus in well-differentiated primary pediatric bronchial epithelial cells. J Virol. 2010;84(22):11718-11728.

55. McDole JR, et al. Goblet cells deliver luminal antigen to CD103+ dendritic cells in the small intestine. Nature. 2012;483(7389):345-349.

56. Townsend MJ, Fallon PG, Matthews DJ, Jolin HE, McKenzie AN. T1/ST2-deficient mice demonstrate the important of T1/ST2 in developing primary T helper cell type 2 responses. J Exp Med. 2000; 191(6):1069-1076

57. Hsu CL, Neilsen CV, Bryce PJ. IL-33 is produced by mast cells and regulates IgE-dependent inflammation. PloS One. 2010;5(8):e11944.

58. Juncadella IJ, et al. Apoptotic cell clearance by bronchial epithelial cells critically influences airway inflammation. Nature. 2013;493(7433):547-551.

59. Hardman CS, Panova V, McKenzie ANJ. IL-33 citrine reporter mice reveal the temporal and spatial expression of IL-33 during allergic lung inflammation. Eur J Immunol. 2012;43(2):1-11.

60. Byers DE, et al. A distinct population of non-B, non-T (NBNT) cells express high levels of IL-13 during acute and chronic airway disease after viral infection. Am J Respir Crit Care Med. 2009;
179:A4293.

61. Byers DE, et al. IL-33-responsive immune cells in the pathogenesis of chronic bronchitis. Am J Respir Crit Care Med. 2013;186:A1004.

62. Polheimer J, et al. Interleukin-33 drives a proinflammatory endothelial activation that selectively targets nonquiescent cells. Arterioscler Thromb Vasc Biol. 2013;33(2):e47-e55.

63. Yagami A, et al. IL-33 mediates inflammatory responses in human lung tissue cells. J Immunol. 2010;185(10):5743-5750.

64. Demehri S, Morimoto M, Holtzman MJ, Kopan R Skin-derived TSLP triggers progression from epidermal-barrier defects to asthma. PLoS Biol. 2009; 7(5):e1000067.

65. Gudbjartsson D, et al. Sequence variants affecting eosinophil numbers associate with asthma and myocardial infarction. Nat Genet. 2009;41(3):342-347.

66. Moffat MF, et al. A large-scale, consortium-based genomewide association study of asthma. NEngJ Med. 2010;363(13):1211-1221

67. Torgerson DG, et al. Meta-analysis of genome-wide association studies of asthma in ethnically diverse North American populations. Nat Genet. 2011; 43(9):887-893.

68. Berndt A, Leme AS, Shapiro SD. Emerging genetics of COPD. EMBO Mol Med. 2012;4(11):1144-1155.

69. Sundlisaeter E, et al. The alarmin interleukin-33 is a notch target in quiescent endothelial cells. $A m J$ Pathol. 2012;181(3):1099-1011.

70. Hogg JC, et al. Survival after lung volume reduction in chronic obstructive pulmonary disease: insights from small airway pathology. Am J Respir Crit Care Med. 2007;176(5):454-459.

71. Miniño AM, Murphy SL, Xu J, Kochanek KD. Deaths: final data for 2008. Natl Vital Stat Rep. 2011; 59(10):1-126

72. WHO. World Health Statistics 2012. Geneva, Switzerland: World Health Organization; 2012.

73. Hautamaki RD, Kobayashi DK, Senior RM, Shapiro SD. Requirement for macrophage elastase for cigarette smoke-induced emphysema in mice. Science. 1997;277(5334):2002-2004.

74. Adair-Kirk TL, et al. Distal airways in mice exposed to cigarette smoke: $\mathrm{Nrf2}$-regulated genes are increased in Clara cells. Am J Respir Cell Mol Biol. 2008; 39(4):400-411.

75. Fallon PG, et al. Identification of an interleukin (IL)-25-dependent cell population that provides IL-4, IL-5, and IL-13 at the onset of helminth expulsion. J Exp Med. 2006;203(4):1105-1116

76. Rickel EA, et al. Identification of functional roles for both IL-17RB and IL-17RA in mediating IL-25-induced activities. J Immunol. 2008;181(6):4299-4310.

77. Palmer $\mathrm{G}$, et al. Inhibition of interleukin-33 signaling attenuates the severity of experimental arthritis. Arthritis Rheum. 2009;60(3):738-749.

78. Verdugo RA, Deschepper CF, Munoz G, Pomp D, Churchill GA. Importance of randomization in microarray experimental designs with Illumina platforms. Nucleic Acids Res. 2009;37(17):5610-5618.

79. Gentleman RC, et al. Bioconductor: open software development for computational biology and bioinformatics. Genome Biol. 2004;5(10):R80.

80. Dunning MJ, Smith ML, Ritchie ME, Tavare S. beadarray: R classes and methods for Illumina beadbased data. Bioinformatics. 2007;23(16):2183-2184.

81. Shi W, Banerjee A, Ritchie ME, Gerondakis S, Smyth GK. Illumina WG-6 BeadChip strips should be normalized separately. BMC Bioinformatics. 2009;10:372.

82. Dunning M, Thorne N, Camilier I, Smith M, Tavaré S. Quality control and low-level statistical analysis of Illumina BeadArrays. Revstat. 2006;4(1):1-30.

83. Cairns JM, Dunning MJ, Ritchie ME, Russell RC, Van den Hurk AF. BASH: a tool for managing BeadArray spatial artefacts. Bioinformatics. 2008; 24(24):2921-2922.

84. Bolstad B, Irizarry R, Astrand M, Speed T. A comparison of normalization methods for high density oligonucleotide array data based on variance and bias. Bioinformatics. 2003;19(2):185-193.

85. Smyth GK. Linear models and empirical Bayes methods for assessing differential expression in microarray experiments. Stat Appl Genet Mol Biol. 2004;3:Article3

86. Yin J, McLoughlin S, Jeffery IB, Glaviano A, Kennedy $B$, Higgins DG. Integrating multiple genome annotation databases improves the interpretation of microarray gene expression data. BMC Genomics. 2010;11:50.

87. Illumina. 2008. Annotation File for Mouse WG6v2, dated 11/9/2008. http://www.switchtoi.com/ annotationfiles.ilmn.

88. Barbosa-Morais NL, et al. A re-annotation pipeline for Illumina BeadArrays: improving the interpretation of gene expression data. Nucleic Acids Res. 2010;38(3):e17.

89. Chen R, Li L, Butte AJ. AILUN: reannotating gene expression data automatically. Nat Methods. 2007; 4(11):879.

90. Thierry-Mieg D, Thierry-Mieg J. AceView: a comprehensive cDNA-supported gene and transcripts annotation. Genome Biol. 2006;7(suppl 1):S12.1-S14.

91. Ballester B, Johnson N, Proctor G, Flicek P. Consistent annotation of gene expression arrays. BMC Genomics. 2010;11:294.

92. Benjamini Y, Hochberg Y. Controlling the false discovery rate - a practical and powerful approach to multiple testing. J R Stat Soc B. 1995;57(1):289-300.

93. Livak KJ, Schmittgen TD. Analysis of relative gene expression data using real-time quantitative PCR and the 2(-Delta Delta C(T)) Method. Methods. 2001; 25(4):402-408.

94. Deslee G, et al. Elastin expression in very severe human COPD. Eur Respir J. 2009;34(2):324-331.

95. You Y, et al. The role of f-box factor foxj 1 in differentiation of ciliated airway epithelial cells. Am J Physiol Lung Cell Mol Physiol. 2004;286(4):L650-L657.

96. Lechner JF, LaVeck MA. A serum-free method for culturing normal human bronchial epithelial cells at clonal density. J Tissue Culture Meth. 1985;9(2):43-48. 